\title{
Enhancement of Quercetin-Induced Apoptosis by Cotreatment with Autophagy Inhibitor Is Associated with Augmentation of BAK-Dependent Mitochondrial Pathway in Jurkat T Cells
}

\author{
Eun Ji Ha, ${ }^{1}$ Ki Yun Kim, ${ }^{1}$ Chae Eun Kim, ${ }^{1}$ Do Youn Jun, ${ }^{2}$ and Young Ho Kim ${ }^{1}$ \\ ${ }^{1}$ Laboratory of Immunobiology, School of Life Science and Biotechnology, College of Natural Sciences, \\ Kyungpook National University, Daegu, Republic of Korea \\ ${ }^{2}$ Astrogen Inc., Techno-Building 313, Kyungpook National University, Daegu 41566, Republic of Korea
}

Correspondence should be addressed to Young Ho Kim; ykim@knu.ac.kr

Received 8 April 2019; Revised 17 July 2019; Accepted 4 September 2019; Published 15 November 2019

Academic Editor: Mario Zoratti

Copyright (@) 2019 Eun Ji Ha et al. This is an open access article distributed under the Creative Commons Attribution License, which permits unrestricted use, distribution, and reproduction in any medium, provided the original work is properly cited.

\begin{abstract}
A flavonoid antioxidant quercetin promotes dose-dependent activation of the ATM-CHK-p53 pathway, downregulation of antiapoptotic survivin, and upregulation of proapoptotic NOXA in human T cell acute lymphoblastic leukemia Jurkat clones (J/Neo and J/BCL-XL). However, the downregulation of antiapoptotic BAG3 and MCL-1 occurred in J/Neo cells but not in J/BCL-XL cells overexpressing BCL-XL. Additionally, several BCL-XL-sensitive intrinsic mitochondrial apoptotic events including apoptotic sub-G $\mathrm{G}_{1}$ cell accumulation, TUNEL-positive DNA fragmentation, BAK activation, mitochondrial membrane potential $(\Delta \psi \mathrm{m})$ loss, caspase-9/caspase-8/caspase-3 activation, and PARP cleavage were induced only in J/Neo cells. Both cytosolic and mitochondrial ROS levels were elevated in quercetin-treated $\mathrm{J} / \mathrm{Neo}$ cells; however, the ROS elevations were almost completely abrogated in J/BCL-XL cells, suggesting the ROS elevations were downstream of BCL-XL-sensitive mitochondrial damage and dysfunction. Wild-type A3, FADD-deficient I2.1, and caspase-8-deficient I9.2 Jurkat clones exhibited similar susceptibilities to the cytotoxicity of quercetin, excluding an involvement of extrinsic pathway in triggering the apoptosis. The autophagic events such as attenuation of AKT-mTOR pathway, formation of acridine orange-stainable acidic vesicular organelles, conversion of microtubule-associated protein 1 light chain 3-I (LC3-I) to LC3-II, and downregulation of p62/SQSTM1 level were detected in quercetin-treated J/Neo and J/BCL-XL cells, regardless of BCL-XL overexpression. Cotreatment with the autophagy inhibitor (3-methyladenine, LY294002, or chloroquine) resulted in a significant enhancement of quercetin-induced BAK activation and subsequently the mitochondrial damage-mediated apoptosis pathway by augmenting the downregulation of BAG3 and MCL-1 levels in J/Neo cells. These results demonstrated that quercetin induces intrinsic apoptosis and cytoprotective autophagy, and autophagy inhibition can potentiate BAK-dependent apoptotic activity of quercetin in Jurkat $\mathrm{T}$ cells.
\end{abstract}

\section{Introduction}

Human T cell acute lymphoblastic leukemia (T-ALL), originating from the malignant transformation of $\mathrm{T}$ cell lineage lymphoblasts, is an aggressive neoplasm and is responsible for approximately $20 \%$ of all ALL cases $[1,2]$. Recent advances in risk-adapted chemotherapy regimens have improved overall survival rates in both childhood and adult T-ALL. However, resistance to chemotherapy and early relapse can occur in T-ALL with high-risk features, leading to unfavorable prognosis and low survival rates [3]. To improve overall survival rates in chemotherapeutic treatment of T-ALL, novel antitumor agents that can minimize drug resistance and side effects are urgently required.

The effectiveness of chemotherapy in tumor regression depends largely on the cytostatic and/or cytotoxic effects of chemotherapeutic drugs on tumor cells. Several biochemical mechanisms, such as apoptosis, necrosis, and autophagy, 
have been implicated in chemotherapy-mediated cytotoxicity toward tumor cells [4-6]. Among these, apoptosis is the most efficient mechanism by which malignant tumor cells can be eliminated following chemotherapy treatment $[7,8]$. In chemotherapy-induced apoptosis of tumor cells, two distinctive death signaling pathways are involved: the intrinsic mitochondria-dependent pathway [9] and the extrinsic death receptor-dependent pathway [10]. The former pathway is mainly associated with tumor cell apoptosis provoked by chemotherapy drugs such as DNA- and microtubuledamaging agents [11].

Autophagy is a cellular catabolic degradation process which is responsible for sequestering unnecessary or dysfunctional cellular components via the formation of doublemembrane vesicles (autophagosomes) and targeting them for degradation via their fusion with lysosomes to generate single-membrane autolysosomes. Several studies have reported that the autophagic capacity is elevated in tumor cells compared to normal cells [12]. This can promote tumor cell survival under stress conditions such as nutritional deprivation and chemotherapy. Because tumor cells can activate autophagy following cellular stress associated with chemotherapy, the pharmacological inhibition of autophagy may be an effective approach to accelerate tumor cell death induced by chemotherapy drugs [13]. However, the role of autophagy activation, which occurs following treatment with chemotherapy drugs, in regulating cancer cell death or survival remains controversial.

Previously, we sought to isolate a pharmacologically safe apoptogenic substance from a collection of edible plants, as edible plant-derived cytotoxic components to tumors may be less toxic to normal cells $[14,15]$. The purification of antitumor ingredients of the grains of Sorghum bicolor (L.) Moench var. Hwanggeumchal, by a serial solvent extraction and silica gel column chromatography, has led to the identification of a flavonoid compound quercetin, which is well-known for its antioxidant and antitumor properties [16-18]. The antitumor effect of quercetin appears to be exerted by cell cycle arrest and apoptotic cell death, which can be mediated by several different mechanisms, including mitochondrial damage- and endoplasmic reticulum (ER) stress-mediated apoptotic pathways. In relation to these quercetin caused activation of apoptotic pathways, topoisomerase II inhibition-mediated DNA damage [19, 20], upregulation of death receptors [21], reactive oxygen species (ROS) generation $[21,22]$, alteration of intracellular $\mathrm{Ca}^{2+}$ via inhibition of sarcoplasmic/endoplasmic reticulum $\mathrm{Ca}^{2+}$ ATPase [23-25], and inhibition of signal transducer and activator of transcription 3 (STAT3) signaling pathway have been implicated [26, 27]. Additionally, quercetin could induce apoptosis and protective autophagy in human malignant glioblastoma U373MG cells [28], human aggressive B cell lymphoma [27], and human bladder cancer cells [29]. However, the individual cellular signaling pathways involved in quercetin-induced apoptosis and autophagy and their interrelationship remain obscure. Moreover, little is known about the involvement of the extrinsic apoptotic pathway in quercetin-induced apoptosis or whether the antitumor activity of quercetin might be applicable to T-ALL.
In this study, we examined whether quercetin, which is one of the most abundant phenolic compounds in S. bicolor grains, could provoke the DNA damage-caused mitochondrial apoptosis pathway and the cytoprotective autophagy pathway simultaneously and sought to identify regulators of crosstalk between these two pathways in quercetin-treated human T-ALL Jurkat cells. Additionally, to examine the involvement of the extrinsic pathway in quercetin-induced mitochondrial apoptosis, we compared apoptotic sub- $\mathrm{G}_{1}$ cell accumulation and $\Delta \psi \mathrm{m}$ loss among quercetin-treated wildtype (A3), Fas-associated death domain- (FADD-) deficient (I2.1), and caspase-8-deficient (I9.2) Jurkat clones.

\section{Materials and Methods}

2.1. Reagents, Chemicals, Cells, and Culture Media. Ethanol (99.9\%) and methanol were obtained from Duksan (Seoul, Korea). Quercetin, 3-methyladenine (3-MA), LY294002 (LY), chloroquine (CQ), dimethyl sulfoxide (DMSO), propidium iodide (PI), 3,3' -dihexyloxacarbocyanine iodide (DiOC6), 4',6-diamidino-2-phenylindole dihydrochloride (DAPI), 3-(4,5-dimethylthiazol-2-yl)-2,5-diphenyl-tetrazolium bromide (MTT), and aminopropyltriethoxy-silane were purchased from Sigma-Aldrich (St. Louis, MO, USA). Dihydroethidium (DHE), a probe specific for detecting reactive oxygen species (ROS), was purchased from Santa Cruz Biotechnology (Santa Cruz, CA, USA). CellROX Deep Red reagent and MitoSOX Red reagent were purchased from Molecular Probes (Carlsbad, CA, USA). The anti-PARP, anti-BAK, anti-BCL-XL, anti-BCL-2, anti-p53, anti-BIM, anti-MCL-1, and anti-p62 antibodies were purchased from Santa Cruz Biotechnology. The anti-p-ATM (Ser-1981), anti-ATM, anti-p-CHK1 (Ser-317), anti-CHK1, anti-pCHK2 (Ser-19), anti-CHK2, anti-p-p53 (Ser-15), anti-pPDK1 (Ser-241), anti-PDK1, anti-p-AKT (Thr-308), antiAKT, anti-p-mTOR (Ser-2448), anti-mTOR, anti-p-ULK (Ser-757), anti-LC3, anti-caspase-8, anti-caspase-9, antcaspase-3, and anti-BID antibodies were obtained from Cell Signaling Technology (Beverly, MA, USA). The anti-BAG3 antibody was purchased from Abcam (Cambridge, UK), and the anti-GAPDH antibody and acridine orange were purchased from Thermo Fisher Scientific (Waltham, USA). The active conformation-specific anti-BAK (Ab-1) antibody was purchased from Calbiochem (San Diego, CA, USA). Human acute leukemia Jurkat T cell clones which were stably transfected with the vector $(\mathrm{J} / \mathrm{Neo})$ or the antiapoptotic $B C L-X L$ gene (J/BCL-XL) were provided by Dr. Dennis Taub (Gerontology Research Center, NIA/NIH, Baltimore, MD, USA). Jurkat $\mathrm{T}$ cell clones A3, I2.1, and I9.2 were purchased from the American Type Culture Collection (Manassas, VA, USA) and maintained in RPMI 1640 complete medium containing 10\% FBS, $20 \mathrm{mM}$ HEPES $(\mathrm{pH}$ 7.0), $50 \mu \mathrm{M} \beta$-mercaptoethanol, and $100 \mu \mathrm{g} / \mathrm{ml}$ gentamicin. For cultivation of both J/Neo and J/BCL-XL cells, $400 \mu \mathrm{g} / \mathrm{ml}$ G418 was added to RPMI 1640 complete medium.

2.2. Extraction and HPLC Analysis of Phenolic Compounds from the Grains of Sorghum bicolor (L.) Moench var. Hwanggeumchal. Ethanol extraction from Sorghum bicolor 
grains was performed as previously described [30], and the dry weights of the $80 \%$ ethanol extract and organic solvent fractions are described in Supplementary Table 1.

The contents of phenolic compounds in the $80 \%$ ethanol extract of $S$. bicolor grains were analyzed by HPLC (Agilent 1200; Agilent Technologies, Waldbronn, Germany) as described elsewhere [31]. Briefly, the analytical column a ZORBAX ODS analytical column $(4.6 \times 250 \mathrm{~mm}$; Agilent Technologies) was used with a guard column (Phenomenex, Torrance, CA, USA). The detection wavelength was set at $280 \mathrm{~nm}$, and the solvent flow rate was held constant at $1.0 \mathrm{ml} / \mathrm{min}$. The mobile phase used for the separation consisted of solvent $\mathrm{A}(0.1 \%$ acetic acid in distilled water) and solvent B ( $0.1 \%$ acetic acid in acetonitrile). A gradient elution procedure was used as $0 \mathrm{~min} 92 \% \mathrm{~A}, 2-27 \mathrm{~min} 90 \% \mathrm{~A}$, $27-50 \min 70 \% \mathrm{~A}, 50-51 \mathrm{~min} 10 \% \mathrm{~A}, 51-60 \mathrm{~min} 0 \% \mathrm{~A}$, and 60-62 min $92 \% \mathrm{~A}$. The injection volume used for analysis was $20 \mu \mathrm{l}$.

The standard phenolic compounds used for HPLC analysis were biochanin A, caffeic acid, p-coumaric acid, $t$-cinnamic acid, gentisic acid, hesperidin, hesperitin, 4-hydroxybenzoic acid, kaempferol, myricetin, naringenin, naringin, protocatechuic acid, quercetin, resveratrol, salicylic acid, and veratric acid (Sigma-Aldrich). The column temperature was set at $60^{\circ} \mathrm{C}$, and the effluent was monitored at $279 \mathrm{~nm}$. All samples were analyzed in triplicates.

Among the 17 phenolic compounds detected in the $80 \%$ ethanol extract, quercetin, kaempferol, naringenin, gentisic acid, salicylic acid, and resveratrol were the most abundant compounds, and their contents were 9.29, 2.90, 2.60, 2.13, 1.76 , and $1.40 \mu \mathrm{g} / \mathrm{mg}$ of the $80 \%$ ethanol extract, respectively (Supplementary Table 2). When the cytotoxic activities of these phenolic compounds were investigated by measuring the $\mathrm{IC}_{50}$ values against human T-ALL Jurkat cell lines (J/Neo and A3), quercetin exhibited the strongest cytotoxicity, followed by kaempferol and resveratrol; however, gentisic acid and naringenin exhibited the lowest cytotoxicity (Supplementary Table 3).

2.3. Cytotoxicity Assay. The cytotoxic effect of individual organic solvent extracts of Sorghum bicolor grains and six major phenolic compounds (quercetin, kaempferol, naringenin, gentisic acid, salicylic acid, and resveratrol) on Jurkat T cells was assessed by the MTT assay as previously described [8]. Briefly, cells $\left(5.0 \times 10^{4} /\right.$ well $)$ were added to a serial dilution of individual samples in 96-well plates (Corning, New York, USA). Following incubation for indicated time periods, MTT solution was added to each well and then incubated for an additional $4 \mathrm{~h}$. The colored formazan crystal generated from MTT was dissolved in DMSO to measure the optical density at $540 \mathrm{~nm}$ by a plate reader.

2.4. Flow Cytometric Analysis. Flow cytometric analyses of apoptotic alterations in the cell cycle status of cells treated with quercetin were performed as previously described [8]. Detection of apoptotic and necrotic cells was performed using an Annexin V-FITC apoptosis kit (Clontech, Takara Bio Inc., Shiga, Japan) as previously described [8]. Quercetin-induced changes in mitochondrial membrane potential $(\Delta \psi \mathrm{m})$ were measured after $\mathrm{DiOC}_{6}$ staining $[32,33]$. Activation of $\mathrm{BAK}$ was detected by the active conformation-specific anti-BAK antibody (Ab-1) [34]. The formation of intracellular acidic vesicular organelles (AVOs) or autolysosome vacuoles was analyzed by flow cytometry with acridine orange staining as previously described [35]. Briefly, cells were incubated with the RPMI 1640 complete medium containing $2 \mu \mathrm{M}$ acridine orange in a $5 \% \mathrm{CO}_{2}$ incubator at $37^{\circ} \mathrm{C}$ for $30 \mathrm{~min}$. Intracellular, cytosolic, and mitochondrial reactive oxygen species (ROS) levels were detected after treatment of the cells with DHE $(1 \mu \mathrm{M})$, CellROX Deep Red reagent $(5 \mu \mathrm{M})$, or MitoSOX Red reagent $(0.5 \mu \mathrm{M})$ in a $\mathrm{CO}_{2}$ incubator at $37^{\circ} \mathrm{C}$ for $30 \mathrm{~min}$, and the fluorescence intensity was analyzed with an Attune NxT Flow Cytometer (Thermo Fisher Scientific, Waltham, MA, USA) as described elsewhere [36].

2.5. TdT-Mediated dUTP Nick-End Labeling (TUNEL) Assay. Vehicle (0.1\% DMSO)-treated control and quercetin-treated cells were adhered onto glass cover slips pretreated with $2 \%$ aminopropyltriethoxy-silane as previously described [34]. The cells were then subjected to fluorescence-TUNEL assay using the In Situ Cell Death Detection Kit (Roche Applied Science, Basel, Switzerland) [36]. To observe the nuclei, the cells were stained with DAPI and examined under the LSM 700 confocal laser scanning microscope (Carl Zeiss MicroImaging GmbH, Germany).

2.6. Preparation of Cell Lysates and Western Blot Analysis. Following cell lysate preparation, an equivalent amount of protein lysate $(20-25 \mu \mathrm{g})$ was electrophoresed on a $4-12 \%$ NuPAGE gradient or 7\% NuPAGE gels with MOPS buffer or Tris-Acetate Buffer and then electrotransferred to a nylon membrane as described elsewhere [8]. Protein detection was performed using an ECL western blot kit (Amersham, Arlington Heights, IL, USA). Densitometry was performed using ImageQuant TL software (Amersham) as previously described [34]. The arbitrary densitometric units for each protein of interest were normalized to the densitometric units for GAPDH.

2.7. Statistical Analysis. Each result is a representative of at least three separate experiments, unless otherwise indicated. Statistical analysis was processed using Student's $t$-test to evaluate the significance of differences between two groups and one-way ANOVA between three or more groups as previously described [36]. $P$ values $<0.05$ were considered significant. Statistical analysis was conducted using the SPSS Statistics version 23 (IBM, Armonk, NY, USA).

\section{Results and Discussion}

3.1. Cytotoxicity of Quercetin in J/Neo and J/BCL-XL Cells. To examine whether the intrinsic mitochondria-dependent apoptosis induction, which can be prevented by BCL-XL overexpression, is crucial for the cytotoxicity of quercetin (Figure 1(a)), the cytotoxic effects of quercetin on $\mathrm{J} / \mathrm{Neo}$ and J/BCL-XL cells were compared. As measured by the MTT assay, the viabilities of $\mathrm{J} / \mathrm{Neo}$ cells in the presence of $12.5,25,50$, and $75 \mu \mathrm{M}$ quercetin were $99.5 \%, 82.5 \%$, $66.9 \%$, and $57.3 \%$, whereas those of J/BCL-XL cells were 
<smiles>O=c1cc(-c2ccc(O)c(O)c2)oc2cc(O)cc(O)c12</smiles>

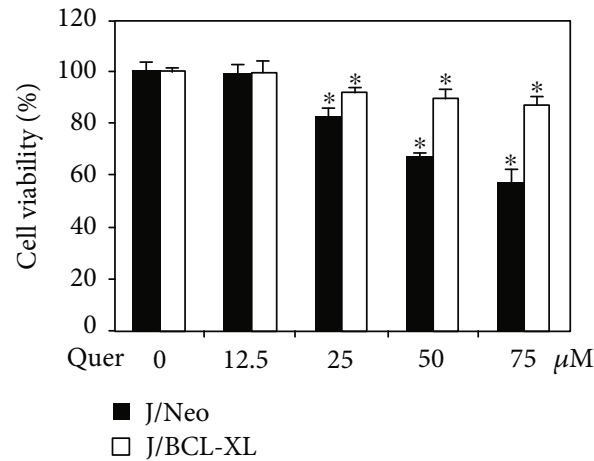

(a)

(b)

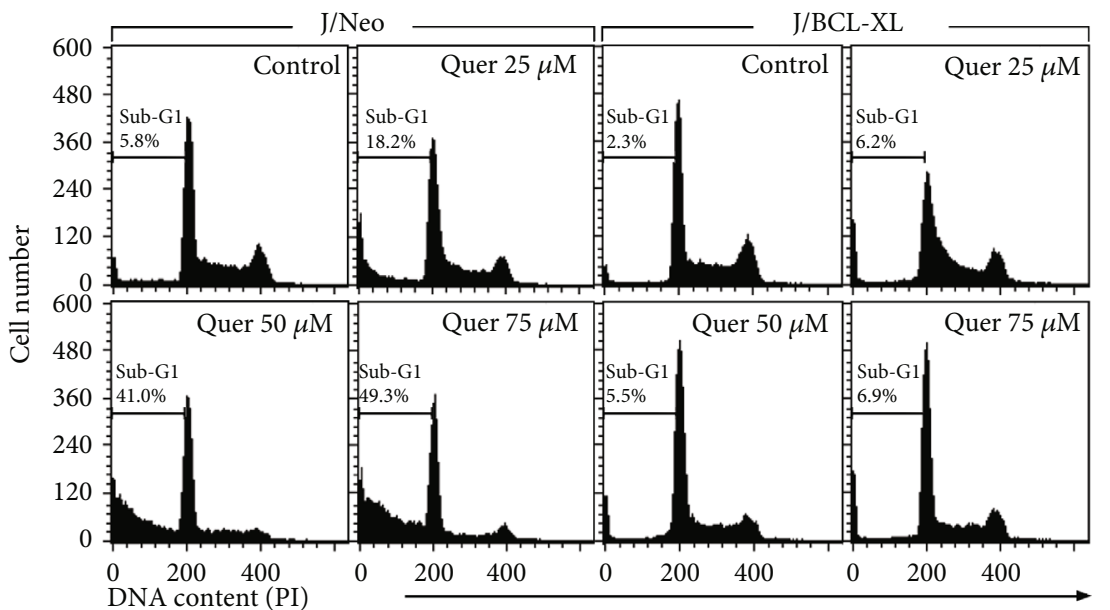

(c)
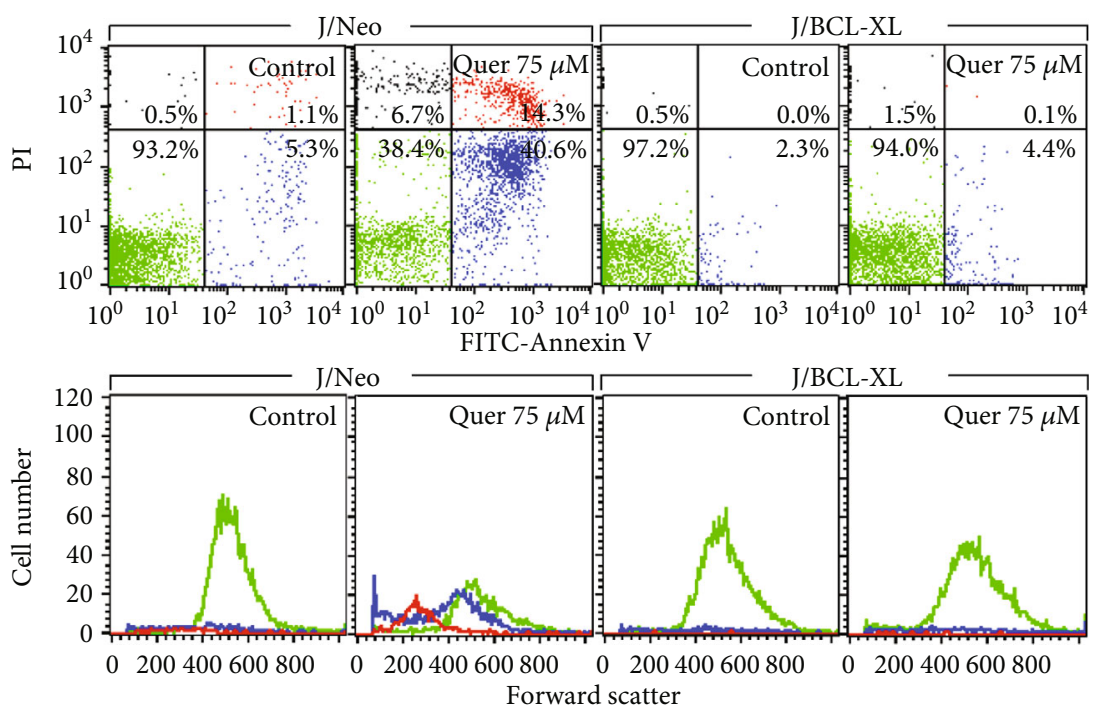

(e)

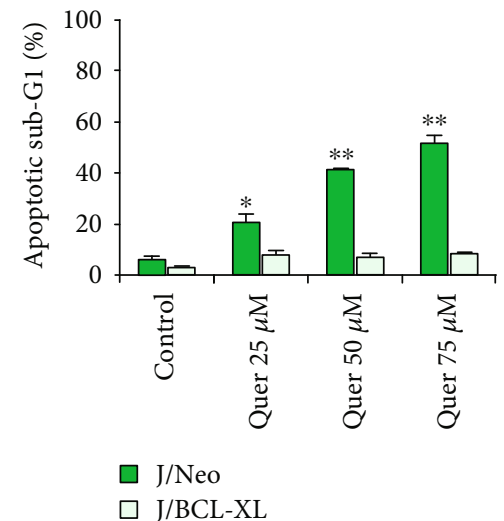

(d)

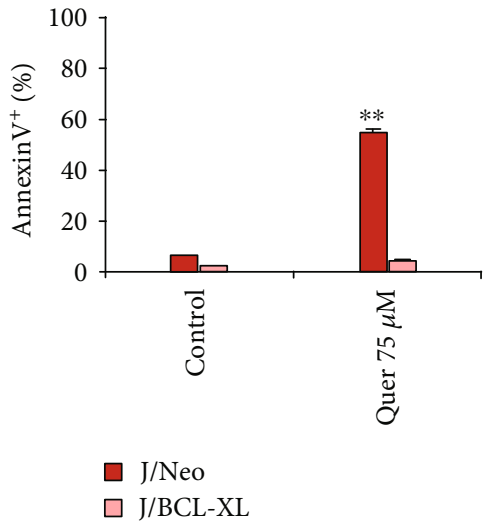

(f)

FIGURE 1: Cytotoxicity of quercetin toward Jurkat T cells was mainly exerted by the induction of BCL-XL-sensitive apoptosis without necrosis. (a) The chemical structure of quercetin. (b) For cell viability analysis, individual cells $\left(5 \times 10^{4} /\right.$ well) were incubated with vehicle $(0.1 \%$ DMSO) or quercetin at indicated doses in a 96-well plate for $11 \mathrm{~h}$ and further incubated with MTT for $4 \mathrm{~h}$ to measure cell viability. Data are expressed as means \pm SD ( $n=3$ with three replicates per independent experiment). (c, d) Cell cycle distribution was measured by flow cytometric analysis with PI staining. (e, f) Annexin V-positive apoptotic cells were determined by flow cytometric analysis with FITC-Annexin V/PI double staining. The forward scatter properties of unstained live, early apoptotic, and late apoptotic cells were measured to analyze alterations in cell size during the induced apoptosis. A representative study is shown and two additional experiments yielded similar results. All data in bar graphs represent the means of triplicate experiments. Error bars represent standard deviations with * and ** indicating $P<0.05$ and $P<0.01$, respectively, compared with the control. 
99.6\%, 91.7\%, 89.8\%, and $88.1 \%$, respectively (Figure $1(\mathrm{~b})$ ). The effect of quercetin on cell cycle distribution of J/Neo and J/BCL-XL cells was compared by flow cytometric analysis. As shown in Figures 1(c) and 1(d), the percentage of sub- $\mathrm{G}_{1}$ cells, which represent apoptotic cells undergoing internucleosomal DNA fragmentation [37], was enhanced in quercetin-treated $\mathrm{J} / \mathrm{Neo}$ cells in a dose-dependent manner; however, the enhancement was not detected in J/BCL-XL cells. These results demonstrated that the cytotoxicity of quercetin was mainly attributed to apoptosis induction which could be completely blocked by BCL-XL overexpression.

During apoptosis induction, cells undergo various morphological changes, including cellular shrinkage and external exposure of phosphatidylserine on the cytoplasmic membrane, whereas necrosis is accompanied by cellular swelling and dilation of organelles, resulting in the plasma membrane ruptures [38]. Previously, it has also been shown that necrotic cells, early apoptotic cells, and late apoptotic cells are different in their FITC-Annexin V/PI dual staining patterns [39]. In these contexts, to elucidate whether quercetin-induced enhancement of the apoptotic sub- $G_{1}$ cell percentage in $\mathrm{J} / \mathrm{Neo}$ cells was caused by apoptosis or apoptosis accompanying necrosis, the cells were analyzed by flow cytometry using FITC-Annexin V and PI staining.

When J/Neo cells were treated with $75 \mu \mathrm{M}$ quercetin for $11 \mathrm{~h}$, the level of necrotic cells (stained with only PI) was negligible (Figures 1(e) and 1(f)). Simultaneously, the early apoptotic cells (stained with only FITC-Annexin V) as well as late apoptotic cells (stained with both FITC-Annexin V and PI) were elevated to the levels of $40.6 \%$ and $14.3 \%$, respectively. Although the unstained live cells following quercetin treatment exhibited no change in their light scattering properties, early and late apoptotic cells showed commonly a decrease in forward scatter, demonstrating typical apoptotic cellular shrinkage rather than necrotic cellular swelling. In contrast, there was no increase in the rate of either early apoptotic or late apoptotic cells in quercetin-treated J/BCL-XL cells. Whereas J/Neo cells following treatment with 25,50 , and $75 \mu \mathrm{M}$ quercetin clearly showed TUNEL-positive nuclei dose-dependently compared with control cells, quercetintreated J/BCL-XL cells failed to show TUNEL-positive cells (Figure 2).

Consequently, these results demonstrated that exposure of Jurkat T cells to quercetin $(25-75 \mu \mathrm{M})$ caused apoptotic cell death in a dose-dependent manner and that the cytotoxicity of quercetin toward Jurkat $\mathrm{T}$ cells could be attributed mainly to BCL-XL-sensitive apoptotic DNA fragmentation, but not to necrosis.

3.2. Induction of the Intrinsic Mitochondrial Apoptosis by Quercetin. Mitochondrial cytochrome $c$ release into the cytosol and subsequent activation of the caspase cascade are frequently associated with chemotherapy-induced apoptosis in tumor cells [40]. Additionally, antiapoptotic proteins (BCL-2, BCL-XL, and MCL-1) are known to protect cells from apoptosis by blocking the activation of proapoptotic BCL-2 family proteins BAK and BAX, which causes permeabilization of the mitochondrial outer membrane, $\Delta \psi \mathrm{m}$ loss, and cytochrome $c$ efflux [41]. To examine the contribution of
BAK/BAX activation and $\Delta \psi \mathrm{m}$ loss to the quercetin-induced apoptosis, we carried out flow cytometric analyses of quercetin-treated cells using active conformation-specific anti-BAK (Ab-1) and anti-BAX (6A7) antibodies for detection of active $\mathrm{BAK}$ and $\mathrm{BAX}$ and using $\mathrm{DiOC}_{6}$ staining for detection of $\Delta \psi \mathrm{m}$ loss. After $\mathrm{J} / \mathrm{Neo}$ cells were treated with quercetin $(25,50$, and $75 \mu \mathrm{M})$ for $11 \mathrm{~h}$, the levels of $\mathrm{J} / \mathrm{Neo}$ cells showing BAK activation appeared to be $13.4 \%, 27.2 \%$, and $34.9 \%$, respectively, whereas the BAK activation was negligible in J/BCL-XL cells (Figures 3(a) and 3(b)). By contrast, the activation of BAX was not detected in either $J / \mathrm{Neo}$ or J/BCL-XL cells after quercetin treatment (data not shown). Under these conditions, quercetin-treated J/Neo cells exhibited $\Delta \psi \mathrm{m}$ loss in a dose-dependent manner; however, the $\Delta \psi \mathrm{m}$ loss was completely abrogated in J/BCL-XL cells (Figures 3(c) and 3(d)). These results demonstrated that quercetin-induced $\Delta \psi \mathrm{m}$ loss was promoted by the BAK activation, which could be blocked by BCL-XL overexpression.

The activation of BAK and resultant $\Delta \psi \mathrm{m}$ loss were reported to be upstream events of the mitochondrial cytochrome $c$ release into the cytosol, mediating the activation of multiple caspases such as caspase-9 and caspase-3 [42]. In this regard, it was highly likely that the activation of caspase- 9 and caspase- 3 , which is a prerequisite for quercetin-induced apoptosis, fails to occur in J/BCL-XL cells due to antiapoptotic action of overexpressed BCL-XL. To test this prediction, we performed western blot analyses to determine whether caspase cascade activation occurred in $\mathrm{J} / \mathrm{Neo}$ cells, but not in J/BCL-XL cells, after quercetin treatment. As shown in Figure 3(e), caspase-9 activation through proteolytic degradation of an inactive procaspase- $9(47 \mathrm{kDa})$ into its active forms $(37 / 35 \mathrm{kDa})$ and caspase- 3 activation through proteolytic degradation of a $32 \mathrm{kDa}$ proenzyme into its $17 \mathrm{kDa}$ active form were detected in quercetin-treated $\mathrm{J} / \mathrm{Neo}$ cells dose-dependently. The activation of caspase- 8 through proteolytic cleavage of proenzyme $(57 \mathrm{kDa})$ into active forms $(43 / 41 \mathrm{kDa})$ was also enhanced in J/Neo cells following quercetin treatment. Simultaneously, the level of BID protein $(22 \mathrm{kDa})$, which is known to be degraded by active caspase- 8 to generate the truncated BID (tBID, $15 \mathrm{kDa}$ ) causing mitochondrial cytochrome $c$ release into the cytosol $[43,44]$, appeared to decrease in quercetintreated J/Neo cells. However, the generation of tBID was not observed by western blot analysis in quercetin-treated $J / N e o$ cells, presumably due to the short half-life of tBID. In agreement with caspase- 3 activation, PARP cleavage was observed in $\mathrm{J} / \mathrm{Neo}$ cells. However, all of these apoptotic responses caused by quercetin treatment were completely abrogated in J/BCL-XL cells.

In relation to antitumor activity of quercetin, inhibition of topoisomerase II and subsequent induction of DNA damage-caused apoptosis were implicated $[19,20]$. Several studies have reported that apoptosis provoked by DNA damage like DNA double-strand break is exerted via induction of the ATM/ATR-CHK1/2-p53 pathway, leading to alterations of proapoptotic and antiapoptotic protein levels [45, 46]. Consistent with these studies, the elevation of phosphorylation state of ATM at Ser-1981, CHK1 at Ser-317, CHK2 at Ser-19, and p53 at Ser-15, which represents their activation 

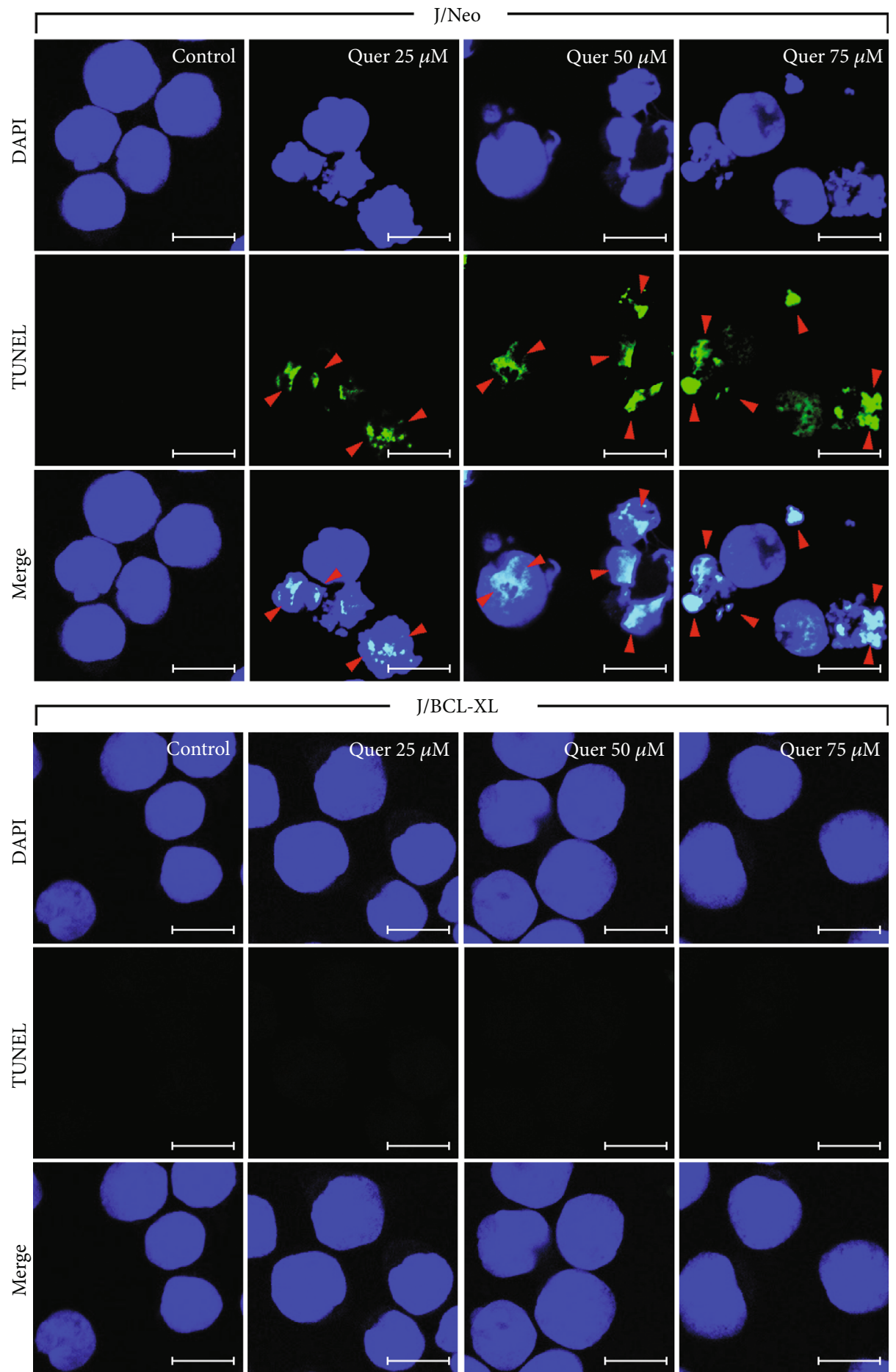

Figure 2: TUNEL-positive DNA fragmentation was observed in quercetin-treated J/Neo cells in a dose-dependent manner but not in J/BCL-XL cells. Individual cells were incubated at a density of $5 \times 10^{5} / \mathrm{ml}$ with vehicle or indicated concentrations of quercetin for $11 \mathrm{~h}$. Apoptotic DNA fragmentation analysis was performed using a TUNEL assay as described in Materials and Methods. Symbols: red arrowhead: TUNEL-positive DNA fragmentation. The scale bar represents a length of $10 \mu \mathrm{m}$ in the images. A representative study is shown; two additional experiments yielded similar results.

possibly resulting from the quercetin-mediated topoisomerase II inhibition and resultant DNA damage, was observed in both $\mathrm{J} / \mathrm{Neo}$ and $\mathrm{J} / \mathrm{BCL}-\mathrm{XL}$ cells treated with quercetin $(25-75 \mu \mathrm{M})$ in a dose-dependent manner (Figure 3(f)). The downstream events of the ATM/ATR-CHK1/2-p53 pathway, upregulation of proapoptotic NOXA level, and downregulation of antiapoptotic survivin level occurred in quercetintreated $\mathrm{J} / \mathrm{Neo}$ and $\mathrm{J} / \mathrm{BCL}-\mathrm{XL}$ cells, whereas antiapoptotic 


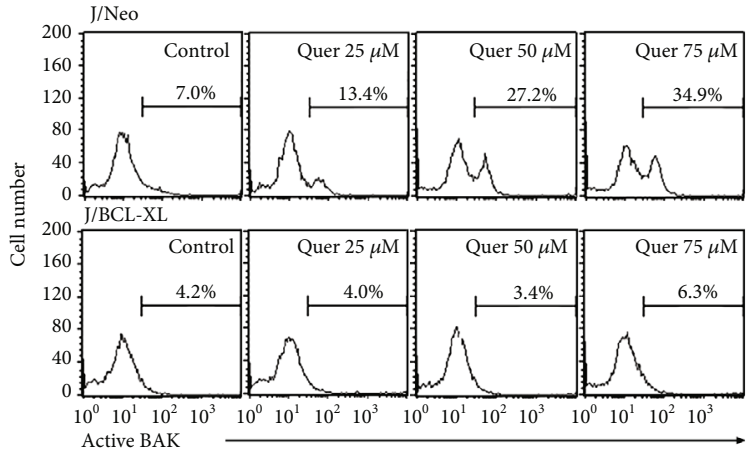

(a)

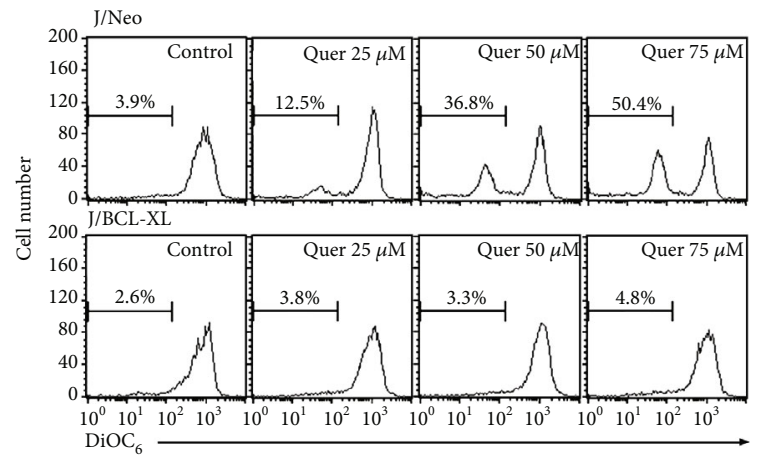

(c)

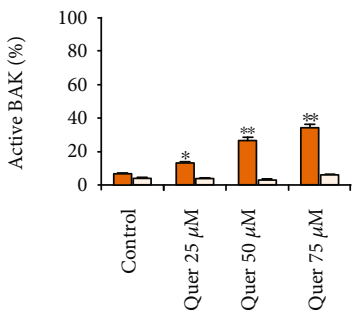

$\square \mathrm{J} / \mathrm{Neo}$ $\square \mathrm{J} / \mathrm{BCL}-\mathrm{XL}$

(b)



(d)

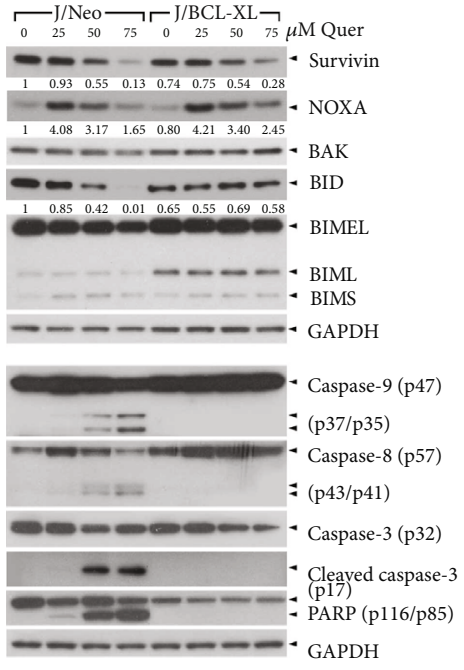

(e)



(f)

FIGURE 3: Quercetin induces mitochondria-dependent apoptotic events and DNA damage-mediated activation of the ATM-CHK-p53 pathway in Jurkat T cells. ( $\mathrm{a}, \mathrm{b})$ BAK activation and (c, d) $\Delta \psi \mathrm{m}$ loss were determined by flow cytometry in J/Neo and J/BCL-XL cells treated with vehicle or quercetin at indicated doses for $11 \mathrm{~h} .(\mathrm{e}, \mathrm{f})$ Total cell lysates from equivalent cultures were prepared, and western blot analyses of survivin, NOXA, BAK, BID, BIM, caspase-9, caspase-8, caspase-3, PARP, p-ATM (Ser-1981), ATM, p-CHK1 (Ser-317), CHK1, p-CHK2 (Ser-19), CHK2, p-p53 (Ser-15), p53, BAG3, BCL-XL, BCL-2, MCL-1, and GAPDH were carried out as described in Materials and Methods. A representative study is shown and two additional experiments yielded similar results. Error bars represent standard deviations with ${ }^{*}$ and ${ }^{* *}$ indicating $P<0.05$ and $P<0.01$, respectively, compared with the control.

BAG3 and MCL-1 levels appeared to decrease only in J/Neo cells. Under these conditions, the BCL-2 and BCL-XL levels were not markedly altered in both cell types following quercetin treatment, as were neither the electrophoretic mobility reduction of BIM during SDS-polyacrylamide gel electropho- resis, exhibiting its activation [34], nor an increase of BAK level. Previously, it was reported that quercetin-mediated downregulation of MCL-1 and survivin rendered nonHodgkin's lymphoma B cells susceptible to TRAIL-induced apoptosis [47]. These previous and current results suggested 
that the proapoptotic impacts of quercetin, which led to the downregulation of cellular MCL-1 and survivin levels, might commonly occur in B lymphoma and T-ALL Jurkat cells.

Consequently, these results indicated that quercetininduced activation of the intrinsic mitochondrial apoptosis pathway was exerted by DNA damage-mediated activation of the ATM/ATR-CHK1/CHK2-p53 pathway and resultant increase of NOXA level and decrease of BAG3, MCL-1, and survivin levels, which predisposed cells to undergo BCL-XL-sensitive BAK activation, $\Delta \psi \mathrm{m}$ loss, and subsequent mitochondria damage-mediated caspase cascade activation.

3.3. Involvement of ROS Generation in Quercetin-Induced Apoptotic Responses. Although quercetin is a well-known flavonoid antioxidant and has been reported to exert cytoprotective roles in oxidative stress-mediated apoptotic conditions $[48,49]$, it has also been shown to cause intracellular ROS generation, which leads to apoptotic cell death in human hepatoma cells [50], human promyelocytic leukemia HL-60 cells [51], and human bladder cancer cells [29]. To examine whether ROS generation is associated with quercetin-induced intrinsic mitochondrial apoptosis in Jurkat T cells, changes in intracellular ROS levels between quercetintreated J/Neo and J/BCL-XL cells were compared by flow cytometry using DHE staining. As shown in Figures 4(a) and $4(\mathrm{~b})$, the mean fluorescent intensity (MFI) value of vehicle-treated J/Neo cells (the control) was 448 , whereas that of quercetin-treated $\mathrm{J} / \mathrm{Neo}$ cells at concentrations of 25, 50, and $75 \mu \mathrm{M}$ was 823,1888 , and 2699 , respectively. Under these conditions, quercetin failed to increase the MFI values in J/BCL-XL cells, demonstrating that the ROS generation in quercetin-treated Jurkat $\mathrm{T}$ cells was targeted by antiapoptotic action of BCL-XL.

The quercetin-caused ROS generation in $\mathrm{J} / \mathrm{Neo}$ and J/BCL-XL cells was further analyzed using the CellROX Red cytosolic ROS indicator [52] and the MitoSOX Red mitochondrial superoxide indicator [53]. Flow cytometric data revealed that quercetin treatment in $\mathrm{J} / \mathrm{Neo}$ cells resulted in a significant elevation in both cytosolic and mitochondrial ROS levels in a dose-dependent manner; however, the quercetin-caused increases in cytosolic and mitochondrial ROS levels were barely or not detected in J/BCL-XL cells (Figures 4(c)-4(f)). As the antiapoptotic action of BCL-XL centers in its blocking of mitochondrial damage to release mitochondrial cytochrome $c$ into the cytosol and thus preventing of cytochrome $c$-mediated caspase cascade activation $[54,55]$, these previous and current results suggested that quercetin-caused intracellular ROS generation might be a downstream event of mitochondrial damage and dysfunction, which can be prevented by overexpressed BCL-XL, rather than a proximal event provoking DNA damageand/or mitochondrial damage-dependent apoptosis.

3.4. Quercetin-Induced Apoptotic Responses in Wild-Type (Clone A3), FADD-Deficient (Clone I2.1), and Caspase-8Deficient (Clone 19.2) Jurkat $T$ Cells. The activation of caspase- 8 plays an essential role as the initiator caspase in the extrinsic death receptor- (DR-) mediated apoptosis signaling pathway [56]. In addition, caspase- 8 activation is known to occur as a downstream event of the intrinsic mitochondrial/caspase-9/caspase-3 apoptosis pathway to comprise a positive feedback loop involving tBID-mediated mitochondrial cytochrome $c$ release in drug-induced apoptosis of tumor cells $[57,58]$. To examine whether the death receptor (DR)/DR ligand system is involved in quercetininduced apoptosis in Jurkat T cells, we compared the apoptotic effect of quercetin on A3 cells with those on I2.1 and I9.2 cells, both of which are known to be refractory to the extrinsic DR-dependent apoptosis [59].

As shown in Figure 5(a), results of western blot analysis showed that although Jurkat A3 appeared to express both FADD and caspase-8, I2.1 and I9.2 cells failed to express FADD and caspase-8, respectively. Following treatment with 50 and $75 \mu \mathrm{M}$ quercetin for $12 \mathrm{~h}$, apoptotic sub- $\mathrm{G}_{1}$ cells were accumulated to a similar extent in A3, I2.1, and I9.2 cells (Figures 5(b) and 5(c)). Simultaneously, the $\Delta \psi \mathrm{m}$ loss induced in A3, I2.1, and I2.1 cells after quercetin treatment was similar to each other (Figures 5(d) and 5(e)). These results confirmed that quercetin-induced apoptosis was provoked by the intrinsic mitochondrial apoptosis pathway, which proceeded independently of the extrinsic apoptosis pathway.

\subsection{Concomitant Induction of Cytoprotective Autophagy and} Apoptosis by Quercetin. In cells under normal conditions, autophagic events are generally suppressed. However, the autophagic response occurs under stress conditions including energy starvation or antitumor drug treatment [60]. As compared to normal counterpart, tumor cells frequently possess an elevated autophagic capacity, allowing tumor cells to be resistant to chemotherapy $[60,61]$. Recently, quercetin has been reported to induce apoptosis and protective autophagy in human cancer cells, such as gastric cancer cells [62], glioblastoma U373MG cells [28], cervical cancer HeLa cells [63], and bladder cancer cells [29]. To further understand the antitumor cytotoxicity of quercetin, the molecular signaling pathways linking quercetin-induced apoptosis and autophagy in J/Neo and J/BCL-XL cells were investigated. Because the acidic vesicular organelles (AVOs) or autolysosome vacuoles are known to be formed by autophagosomelysosome fusion as a key feature of autophagy [64], flow cytometric analyses were carried out to detect if the formation of AVOs or autolysosome vacuoles is elevated in quercetin-treated J/Neo and J/BCL-XL cells.

As shown in Figures 6(a) and 6(b), while acridine orange could effectively stain the cellular DNA/RNA in green (AO-Green) and the AVOs or autolysosome vacuoles in red (AO-Red), a significant dose-dependent enhancement of the $\mathrm{AO}$ red fluorescence was observed in quercetin-treated (25 and $50 \mu \mathrm{M}$ ) J/Neo and J/BCL-XL cells, suggesting that the AVOs or autolysosome vacuoles were formed by quercetin irrespective of the presence of overexpressed BCL-XL. At the same time, treatment of $\mathrm{J} / \mathrm{Neo}$ cells with 25 and $50 \mu \mathrm{M}$ quercetin resulted in the accumulation of low AO-Green/low AO-Red cells to the levels of $21.3 \%$ and $41.7 \%$, respectively; however, the increase of low AO-Green/low AO-Red cells was not or barely detected in J/BCL-XL cells. The percentages of these subpopulations, which are characterized by low 




(a)

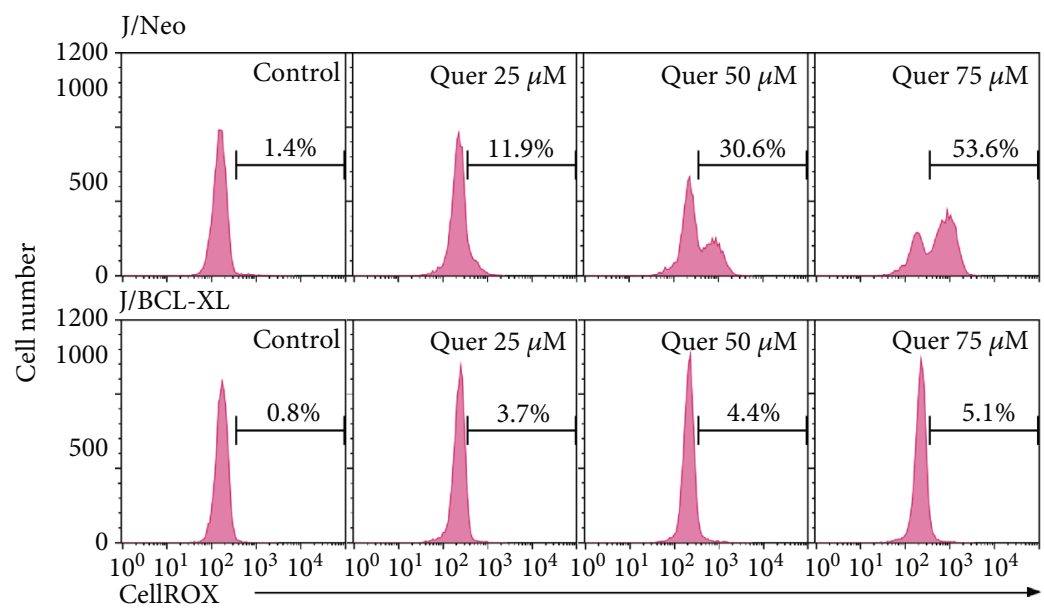

(c)

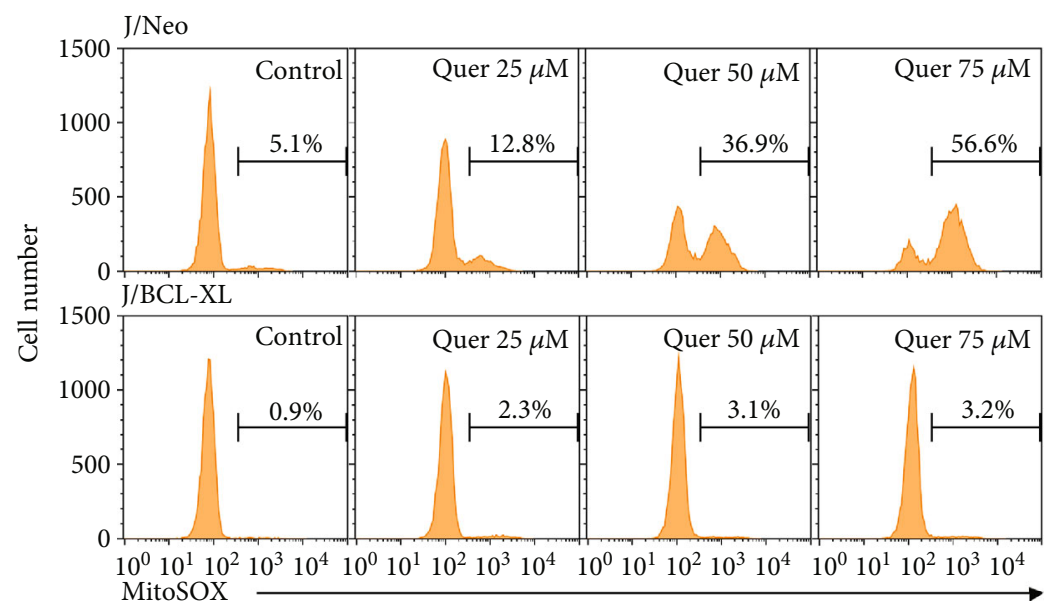

(e)

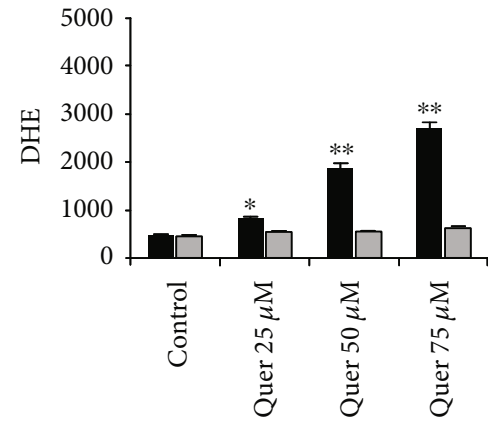

$\mathrm{J} / \mathrm{Neo}$

J/BCL-XL

(b)

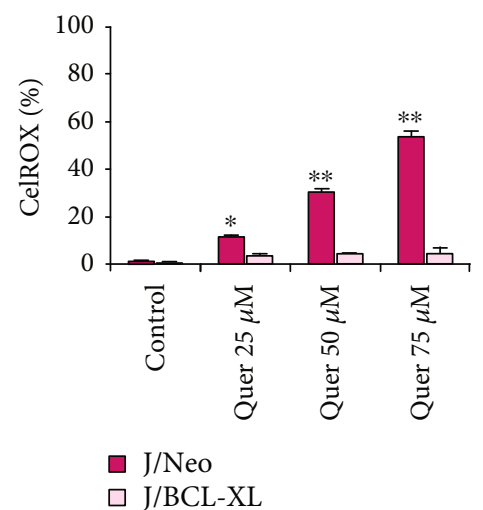

(d)

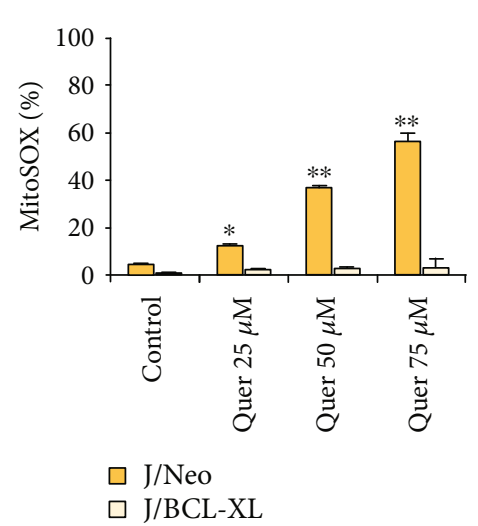

(f)

FIGURE 4: Cytosolic and mitochondrial ROS levels were commonly enhanced in quercetin-treated J/Neo cells but not in J/BCL-XL cells. $(\mathrm{a}, \mathrm{b})$ The intracellular ROS, (c, d) cytosolic ROS, and (e, f) mitochondrial ROS levels in J/Neo and J/BCL-XL cells treated with vehicle or quercetin at indicated doses for $11 \mathrm{~h}$ were analyzed using flow cytometry with DHE, CellROX Deep Red, and MitoSOX Red staining, respectively, and indicated by the MFI or percentage of the cells. A representative study is shown and two additional experiments yielded similar results. Error bars represent standard deviations with * and ${ }^{* *}$ indicating $P<0.05$ and $P<0.01$, respectively, compared with the control. 




(a)

(b)

(c)

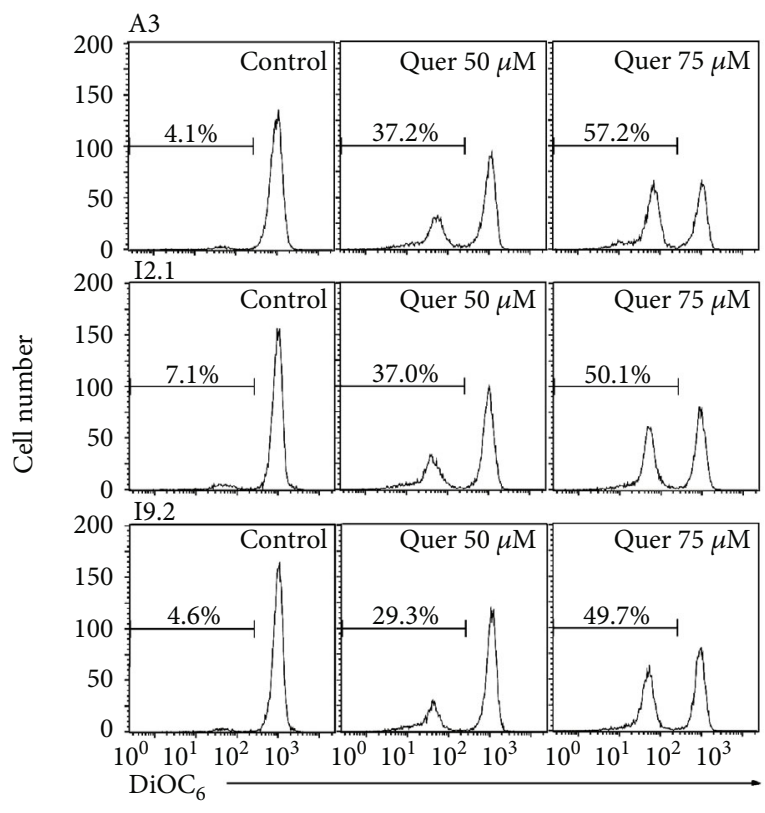

(d)

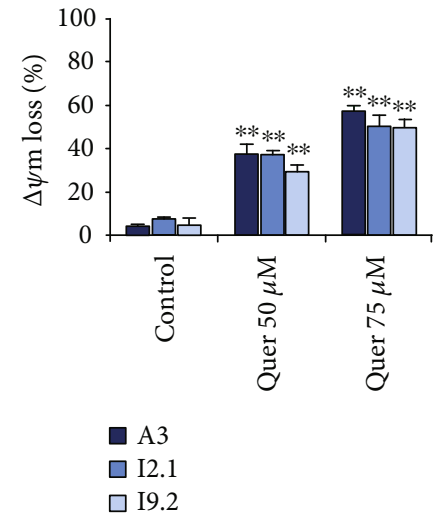

(e)

FIGURE 5: The sensitivity of FADD- and capase-8-positive wild-type Jurkat clone A3 to the apoptogenic activity of quercetin was similar to that of FADD-deficient Jurkat T cell clone I2.1 and caspase-8-deficient Jurkat T cell clone I9.2. (a) Exponentially growing individual cells were subjected to western blot analyses of caspase-8, FADD, and GAPDH as described in Materials and Methods. (b-e) After A3, I2.1, or I9.2 cells $\left(5 \times 10^{5}\right.$ cells $\left./ \mathrm{ml}\right)$ were incubated with vehicle or quercetin at indicated doses for $11 \mathrm{~h}$, the cells were stained with PI and with $\mathrm{DiOC}_{6}$ for flow cytometric analysis of the cell cycle state and $\Delta \psi \mathrm{m}$ loss, respectively, as described in Materials and Methods. A representative study is shown and two additional experiments yielded similar results. Error bars represent standard deviations with ${ }^{* *}$ indicating $P<0.01$, compared with the control.

AO-Green/low AO-red fluorescence, were similar to the percentages of apoptotic sub- $\mathrm{G}_{1}$ cells detected by flow cytometric analysis with PI staining (data not shown). This suggested that the low AO-Green/low AO-Red cells might be late apoptotic cells possessing apoptotic DNA fragmentation and impairments in AO-stainable acidic compartments.
Fluorescence microscopy also exhibited a remarkable elevation in quercetin-induced formation of AVOs in $\mathrm{J} / \mathrm{Neo}$ and J/BCL-XL cells (Figure 6(c)). Under these conditions, western blot analysis revealed that the autophagy-associated alteration of two autophagy marker proteins, such as conversion of microtubule-associated protein 1 light chain 3-I 


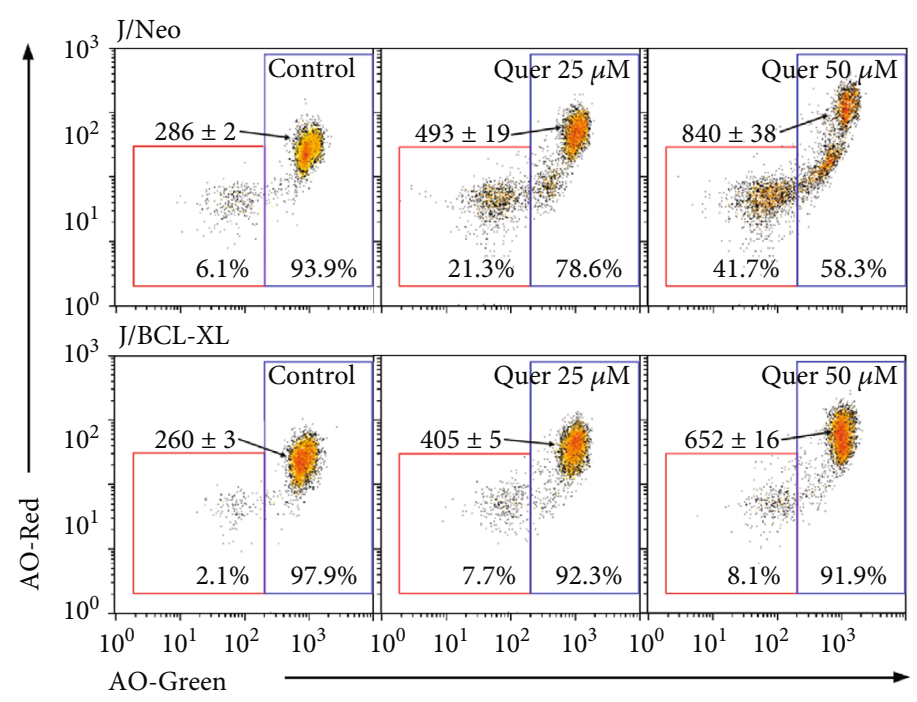

(a)

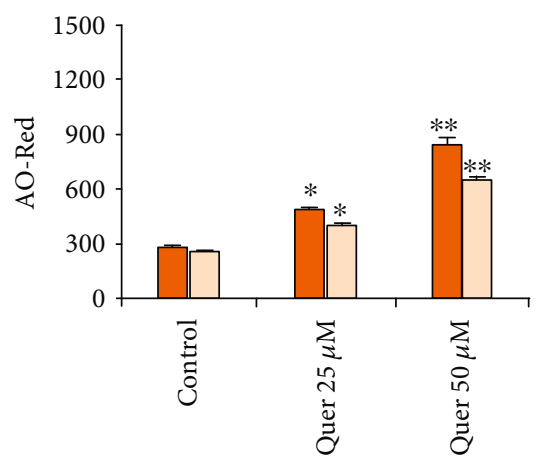

$\square \mathrm{J} / \mathrm{Neo}$

$\square \mathrm{J} / \mathrm{BCL}-\mathrm{XL}$

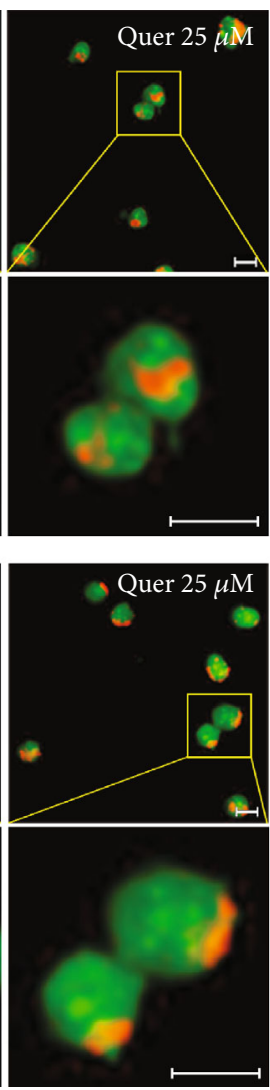

(c)
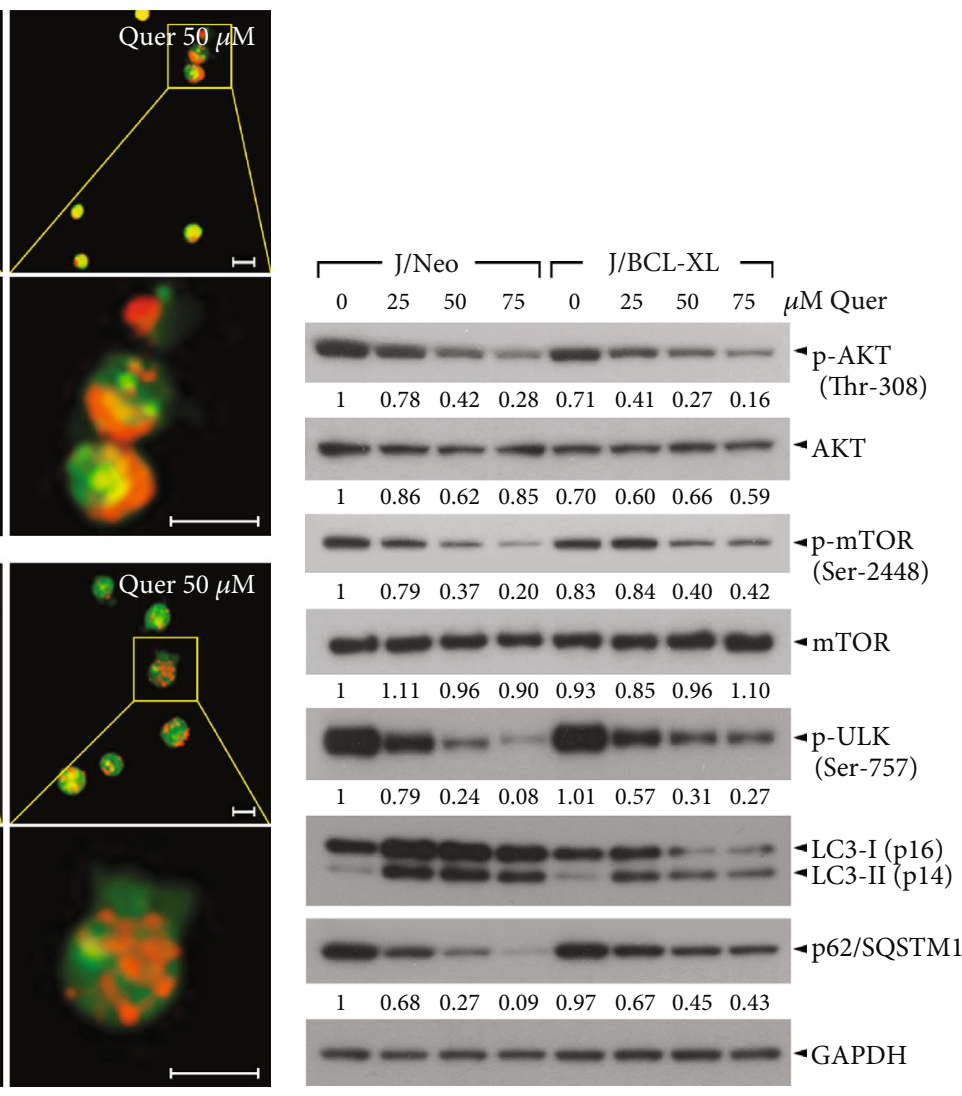

(d)

FIGURE 6: Quercetin induces autophagy in J/Neo and J/BCL-XL cells. (a-c) After cells were treated with vehicle or quercetin at indicated doses $(25$ and $50 \mu \mathrm{M})$ for $11 \mathrm{~h}$, the enhancement of acridine orange- (AO-) Red fluorescence was analyzed by flow cytometry and fluorescence microscopy, respectively. The AO-Red fluorescence indicates the formation of AVOs and autolysosome vacuoles, resulting from autophagy induction, and the AO-Green fluorescence indicates AO staining of DNA/RNA in cells. Error bars represent standard deviations with ${ }^{*}$ and ${ }^{* *}$ indicating $P<0.05$ and $P<0.01$, respectively, compared with the control. (d) Western blot analyses of p-AKT (Thr-308), AKT, p-mTOR (Ser-2448), mTOR, p-ULK (Ser-757), LC3-I/LC3-II, p62/SQSTM1, and GAPDH were performed as described in Materials and Methods. A representative study is shown and two additional experiments yielded similar results.

(LC3-I, $16 \mathrm{kDa})$ into LC3-II $(14 \mathrm{kDa})$ and downregulation of the polyubiquitin-binding protein p62/sequestosome 1 (p62/SQSTM1) level, was induced in quercetin-treated
J/Neo and J/BCL-XL cells (Figure 6(d)). Furthermore, AKT-mTOR pathway inhibition, as evidenced by reduction in the levels of p-AKT (Thr-308), p-mTOR (Ser-2448), and 


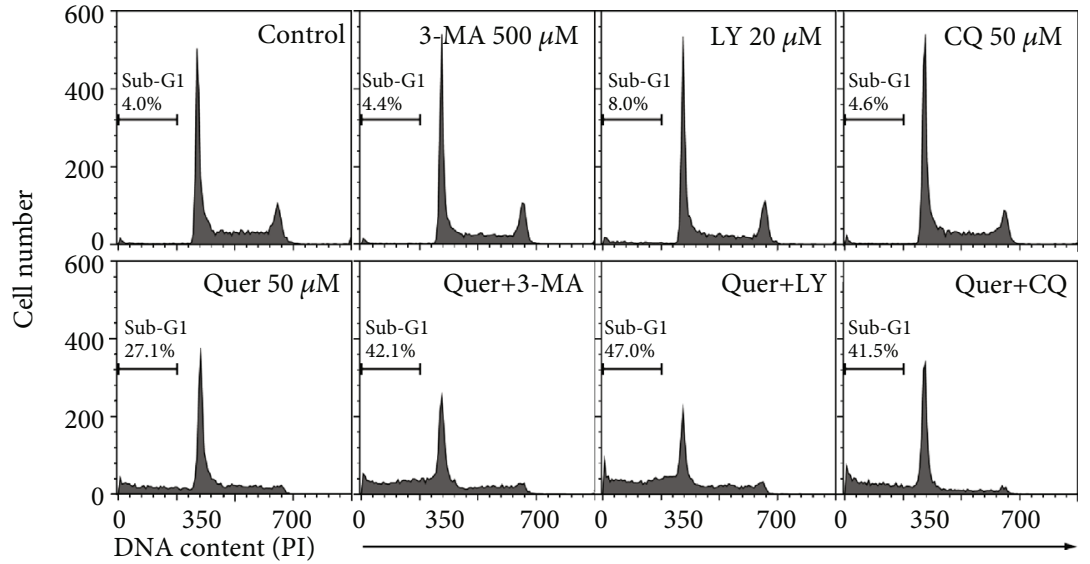

(a)

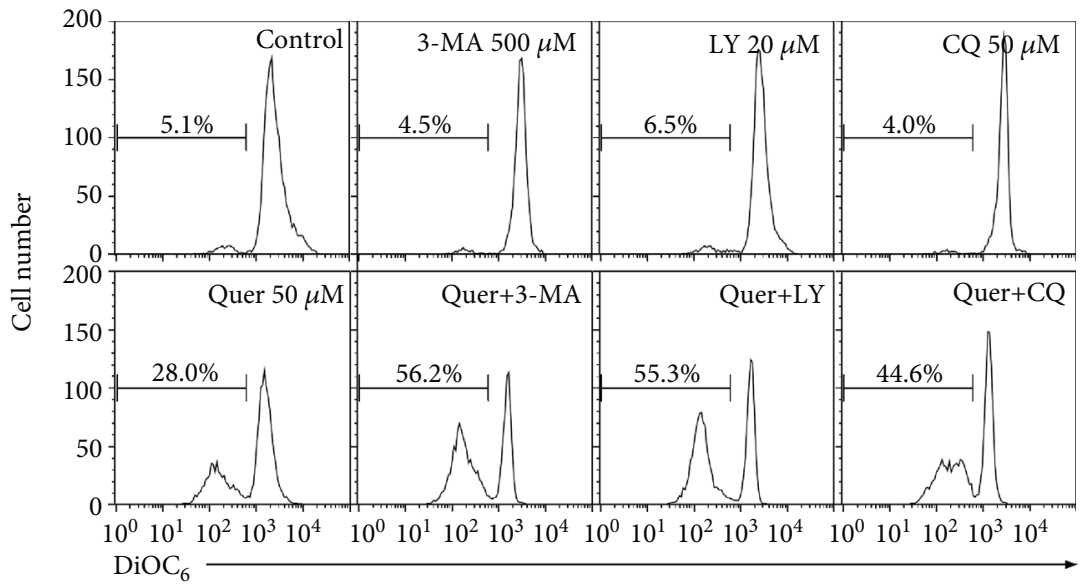

(c)

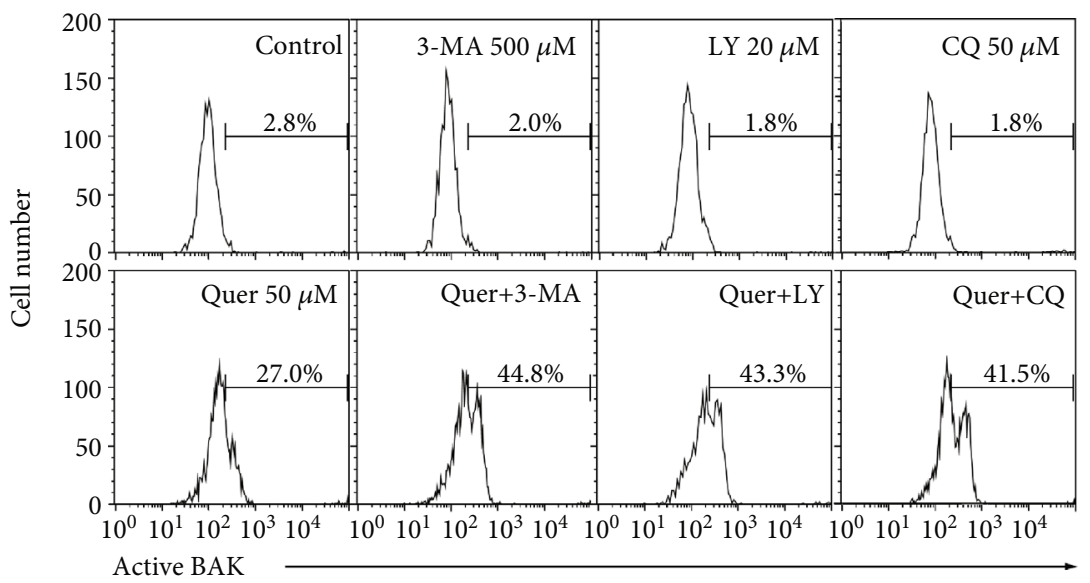

(e)



(b)

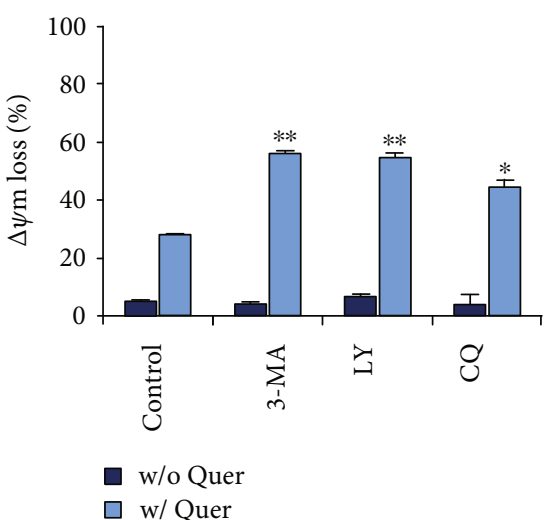

(d)

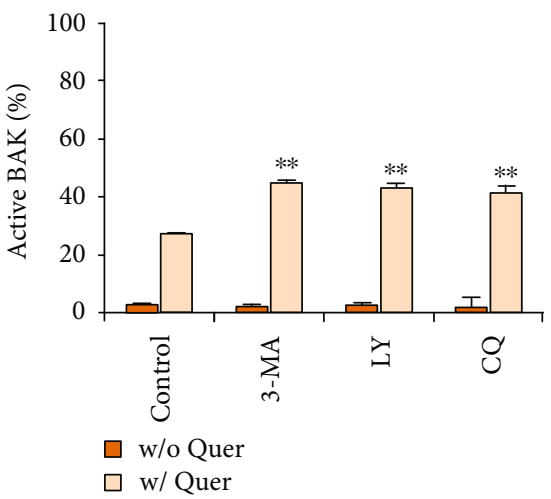

(f)

FIGURE 7: Cotreatment of J/Neo cells with pharmacological inhibitors (3-MA, LY, or CQ) of autophagy promotes quercetin-induced sub-G cell accumulation, $\Delta \psi \mathrm{m}$ loss, and BAK activation. (a-f) After treatment with $50 \mu \mathrm{M}$ quercetin in the absence or presence of either $500 \mu \mathrm{M} 3-\mathrm{MA}, 20 \mu \mathrm{M} \mathrm{LY}$, or $50 \mu \mathrm{M}$ CQ for $7 \mathrm{~h}$, the cells were harvested and subjected to flow cytometry to analyze the percentage of apoptotic sub-G $\mathrm{G}_{1}$ cells, $\Delta \psi \mathrm{m}$ loss, and BAK activation. A representative study is shown and two additional experiments yielded similar results. Error bars represent standard deviations with * and ${ }^{* *}$ indicating $P<0.05$ and $P<0.01$, respectively, compared with the control.

p-ULK (Ser-757), was observed to a similar level in both cell types following quercetin treatment. These results indicated that quercetin caused autophagy via suppression of the AKT-mTOR pathway in Jurkat $\mathrm{T}$ cells, regardless of the overexpression of BCL-XL. It is noteworthy that because the AKT pathway is also known to play an important role in cell survival by mediating inhibitory phosphorylation of proapoptotic protein $\mathrm{BAD}$, it cannot exclude a possible 


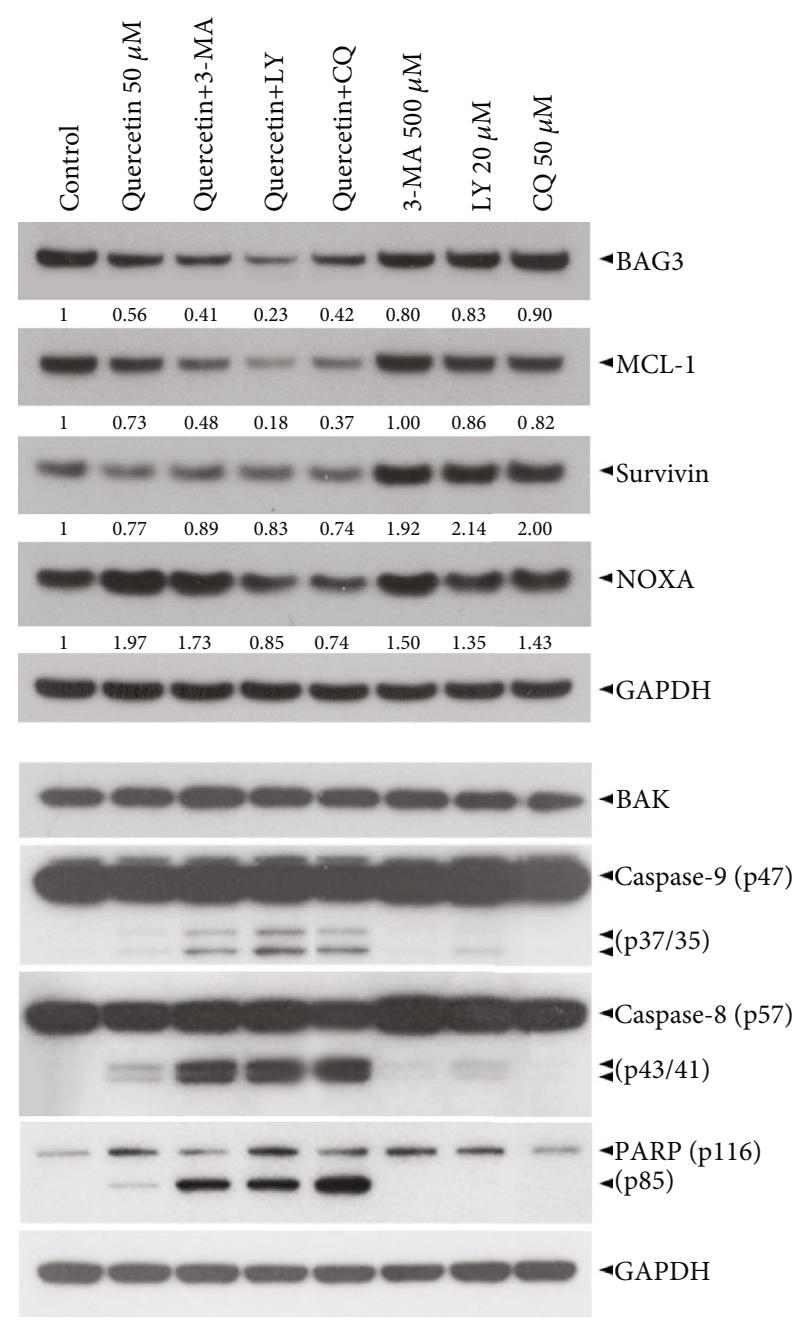

(a)

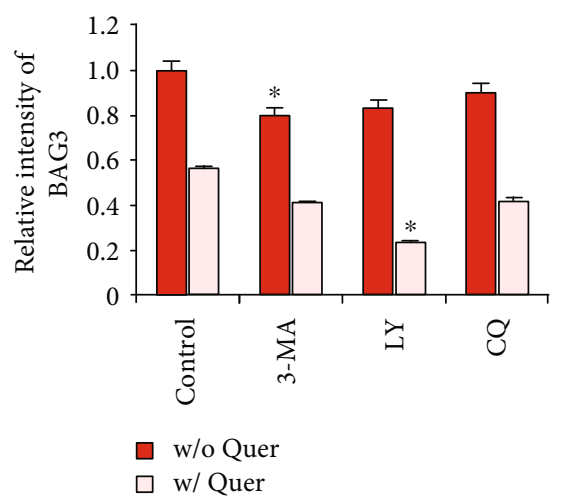

(b)

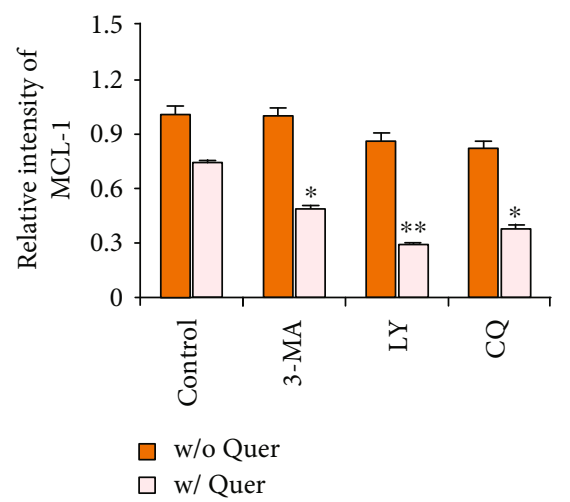

(c)

FIGURE 8: Cotreatment of J/Neo cells with pharmacological inhibitors (3-MA, LY, or CQ) of autophagy promotes quercetin-induced downregulation of BAG3 and MCL-1 levels, caspase-9/caspase- 8 activation, and PARP cleavage. (a) After treatment with $50 \mu \mathrm{M}$ quercetin in the absence or presence of either $500 \mu \mathrm{M} 3-\mathrm{MA}, 20 \mu \mathrm{M} \mathrm{LY}$, or $50 \mu \mathrm{M}$ CQ for $7 \mathrm{~h}$, the cells were harvested and subjected to western blot analyses of BAG3, MCL-1, survivin, NOXA, BAK, caspase-9, caspase-8, PARP, and GAPDH as described in Materials and Methods. A representative study is shown and two additional experiments yielded similar results. (b, c) The arbitrary densitometric units of BAG3 and MCL-1 were normalized to those of GAPDH. Error bars represent standard deviations with ${ }^{*}$ and ${ }^{* *}$ indicating $P<0.05$ and $P<0.01$, respectively, compared with the control.

contribution of the AKT inhibition, causing promotion of apoptosis, in quercetin-induced cell death $[26,65,66]$.

To examine the relationship between quercetin-induced autophagy and apoptosis, the effect of the pharmacologic inhibitors of autophagy on quercetin-induced sub- $\mathrm{G}_{1}$ cell accumulation and $\Delta \psi \mathrm{m}$ loss was investigated in $\mathrm{J} / \mathrm{Neo}$ cells. The autophagy inhibitors 3-methyladenine (3-MA) and LY294002 (LY) can inhibit the class III PI3K activity required for the formation of autophagosomes during autophagy process [67], whereas the autophagy inhibitor chloroquine (CQ) blocks autolysosome formation in the late stage of autophagy [68]. After treatment of $\mathrm{J} / \mathrm{Neo}$ cells with $50 \mu \mathrm{M}$ quercetin for $7 \mathrm{~h}$, the induced apoptotic sub- $\mathrm{G}_{1}$ peak and $\Delta \psi \mathrm{m}$ loss were $27.1 \%$ and $28.0 \%$, respectively (Figures $7(\mathrm{a})-7(\mathrm{~d})$ ). The quercetin-induced apoptotic sub- $G_{1}$ peak was enhanced to the levels of $42.1 \%, 47.0 \%$, and $41.5 \%$ in the presence of $500 \mu \mathrm{M} 3-\mathrm{MA}, 20 \mu \mathrm{M} \mathrm{LY}$, and $50 \mu \mathrm{M} \mathrm{CQ}$, respectively. The quercetin-induced $\Delta \psi \mathrm{m}$ loss was also elevated to the levels of $56.2 \%, 55.3 \%$, and $44.6 \%$ by $500 \mu \mathrm{M} 3-\mathrm{MA}, 20 \mu \mathrm{M} \mathrm{LY}$, and $50 \mu \mathrm{M} \mathrm{CQ}$, respectively. Additionally, quercetininduced BAK activation was markedly enhanced by 3-MA, LY, or CQ (Figures 7(e) and 7(f)). Furthermore, results of western blot analyses revealed that quercetin-induced apoptotic events, including reduction of BAG3 and MCL-1 levels, caspase-9/caspase-8 activation, and PARP cleavage, were markedly elevated in the presence of 3-MA, LY294002, or CQ in common (Figures 8(a)-8(c)). However, the level of BAK protein remained constant.

Consequently, these results demonstrate that the autophagic events provoked in $\mathrm{J} / \mathrm{Neo}$ cells being treated with 
quercetin were a cellular response to sustain cell survival from mitochondrial damage-mediated apoptotic cell death via reducing the quercetin-induced BAK activation.

\section{Conclusion}

This study demonstrated that the cytotoxic effect of quercetin $(25-75 \mu \mathrm{M})$ on Jurkat T cells was exerted by inducing intrinsic mitochondrial apoptosis via sequentially mediating the activation of the ATM-CHK1/CHK2-p53 pathway, upregulation of proapoptotic NOXA level, downregulation of antiapoptotic BAG3, MCL-1, and survivin levels, BAK activation, $\Delta \psi \mathrm{m}$ loss, ROS generation, caspase cascade activation, PARP cleavage, and apoptotic DNA fragmentation. These apoptogenic actions of quercetin, except for upstream events of the mitochondrial damage-dependent apoptosis pathway, are completely blocked by the overexpression of BCL-XL. Quercetin induces autophagy resulting from attenuating the AKT-mTOR pathway, leading to the manifestation of several cellular autophagy markers including increased formation of acridine orange-stainable AVOs, LC3-I/LC3-II conversion, and p62/SQSTM1 downregulation. However, none of these quercetin-caused autophagic events are abrogated by BCL-XL overexpression. Cotreatment with a pharmacological autophagy inhibitor (3-MA, LY, or CQ) enhances the quercetin-induced reduction of BAG3 and MCL-1 levels, BAK activation, and subsequent promotion of mitochondrial damage-dependent apoptosis. These results suggested that combining quercetin with an autophagy inhibitor may be a promising antitumor treatment of human T-ALL.

\section{Data Availability}

The data used to support the findings of this study are included within the article.

\section{Conflicts of Interest}

The authors declare no conflict of interest.

\section{Acknowledgments}

This work was performed with the support of "Cooperative Research Program for Agriculture Science \& Technology Development (Project No. PJ006638)" Rural Development Administration, Republic of Korea.

\section{Supplementary Materials}

Supplementary Table 1: dry weights of the 95\% ethanol extract and its organic solvent fractions prepared from the grains of Sorghum bicolor (L.) Moench var. Hwanggeumchal. Supplementary Table 2: HPLC-dependent analysis of phenolic components in 95\% EtOH extract and its organic solvent fractions from the grains of Sorghum bicolor (L.) Moench var. Hwanggeumchal. Supplementary Table 3: inhibitory effect of the major phenolic compounds in organic solvent fractions prepared from the grains of Sorghum bicolor (L.) Moench var. Hwanggeumchal on proliferation of human T-ALL Jurkat cells. Graphic abstract. (Supplementary Materials)

\section{References}

[1] I. Aifantis, E. Raetz, and S. Buonamici, "Molecular pathogenesis of T-cell leukaemia and lymphoma," Nature Reviews Immunology, vol. 8, no. 5, pp. 380-390, 2008.

[2] P. Van Vlierberghe and A. Ferrando, "The molecular basis of T cell acute lymphoblastic leukemia," Journal of Clinical Investigation, vol. 122, no. 10, pp. 3398-3406, 2012.

[3] S. P. Hunger, X. Lu, M. Devidas et al., "Improved survival for children and adolescents with acute lymphoblastic leukemia between 1990 and 2005: a report from the children's oncology group," Journal of Clinical Oncology, vol. 30, no. 14, pp. 1663$1669,2012$.

[4] R. K. Amaravadi and C. B. Thompson, "The roles of therapy-induced autophagy and necrosis in cancer treatment," Clinical Cancer Research, vol. 13, no. 24, pp. 7271-7279, 2007.

[5] S. Mansilla, L. Llovera, and J. Portugal, "Chemotherapeutic targeting of cell death pathways," Anti-Cancer Agents in Medicinal Chemistry, vol. 12, no. 3, pp. 226-238, 2012.

[6] M. S. Ricci and W. X. Zong, "Chemotherapeutic approaches for targeting cell death pathways," The Oncologist, vol. 11, no. 4, pp. 342-357, 2006.

[7] S. H. Kaufmann and W. C. Earnshaw, "Induction of apoptosis by cancer chemotherapy," Experimental Cell Research, vol. 256, no. 1, pp. 42-49, 2000.

[8] D. Y. Jun, J. S. Kim, H. S. Park et al., “Apoptogenic activity of auraptene of Zanthoxylum schinifolium toward human acute leukemia Jurkat T cells is associated with ER stress-mediated caspase- 8 activation that stimulates mitochondria-dependent or -independent caspase cascade," Carcinogenesis, vol. 28, no. 6, pp. 1303-1313, 2007.

[9] S. Fulda and K. M. Debatin, "Extrinsic versus intrinsic apoptosis pathways in anticancer chemotherapy," Oncogene, vol. 25, no. 34, pp. 4798-4811, 2006.

[10] C. Friesen, I. Herr, P. H. Krammer, and K. M. Debatin, "Involvement of the CD95 (APO-1/Fas) receptor/ligand system in drug-induced apoptosis in leukemia cells," Nature Medicine, vol. 2, no. 5, pp. 574-577, 1996.

[11] S. Desagher and J. C. Martinou, "Mitochondria as the central control point of apoptosis," Trends in Cell Biology, vol. 10, no. 9, pp. 369-377, 2000.

[12] K. Degenhardt, R. Mathew, B. Beaudoin et al., "Autophagy promotes tumor cell survival and restricts necrosis, inflammation, and tumorigenesis," Cancer Cell, vol. 10, no. 1, pp. 51-64, 2006.

[13] J. Huang and D. J. Klionsky, "Autophagy and human disease," Cell Cycle, vol. 6, no. 15, pp. 1837-1849, 2007.

[14] A. Amin, H. Gali-Muhtasib, M. Ocker, and R. SchneiderStock, "Overview of major classes of plant-derived anticancer drugs," International Journal of Biomedical Sciences, vol. 5, no. 1, pp. 1-11, 2009.

[15] E. Safarzadeh, S. S. Shotorbani, and B. Baradaran, "Herbal medicine as inducers of apoptosis in cancer treatment," Advanced Pharmaceutical Bulletin, vol. 4, suppl 1, pp. 421427, 2014.

[16] G. Williamson, G. W. Plumb, Y. Uda, K. R. Price, and M. J. C. Rhodes, "Dietary quercetin glycosides: antioxidant activity and induction of the anticarcinogenic phase II marker enzyme quinone reductase in Hepalclc7 cells," Carcinogenesis, vol. 17, no. 11, pp. 2385-2387, 1996. 
[17] M. S. Kim, I. T. Oh, D. Y. Jun et al., "Anticoagulant and fibrinolytic activities of Hwanggeumchal sorghum in vitro," Journal of Life Science, vol. 23, no. 12, pp. 1460-1470, 2013.

[18] S. Y. Zheng, Y. Li, D. Jiang, J. Zhao, and J. F. Ge, “Anticancer effect and apoptosis induction by quercetin in the human lung cancer cell line A-549," Molecular Medicine Reports, vol. 5, no. 3, pp. 822-826, 2012.

[19] G. Cantero, C. Campanella, S. Mateos, and F. Cortés, “Topoisomerase II inhibition and high yield of endoreduplication induced by the flavonoids luteolin and quercetin," Mutagenesis, vol. 21, no. 5, pp. 321-325, 2006.

[20] Z. Zhang, S. Chen, H. Mei et al., "Ginkgo biloba leaf extract induces DNA damage by inhibiting topoisomerase II activity in human hepatic cells," Scientific Reports, vol. 5, no. 1, 2015.

[21] L. Yi, Y. Zongyuan, G. Cheng, Z. Lingyun, Y. Guilian, and G. Wei, "Quercetin enhances apoptotic effect of tumor necrosis factor-related apoptosis-inducing ligand (TRAIL) in ovarian cancer cells through reactive oxygen species (ROS) mediated CCAAT enhancer-binding protein homologous protein (CHOP)-death receptor 5 pathway," Cancer Science, vol. 105, no. 5, pp. 520-527, 2014.

[22] Y.-S. Ma, C.-N. Yao, H.-C. Liu et al., "Quercetin induced apoptosis of human oral cancer SAS cells through mitochondria and endoplasmic reticulum mediated signaling pathways," Oncology Letters, vol. 15, no. 6, pp. 9663-9672, 2018.

[23] V. Shoshan and D. H. MacLennan, "Quercetin interaction with the $\left(\mathrm{Ca}^{2+}+\mathrm{Mg}^{2+}\right)$-ATPase of sarcoplasmic reticulum," The Journal of Biological Chemistry, vol. 256, no. 2, pp. 887892, 1981.

[24] O. A. Ogunbayo and F. Michelangeli, "Related flavonoids cause cooperative inhibition of the sarcoplasmic reticulum $\mathrm{Ca}^{2+}$ ATPase by multimode mechanisms," FEBS Journal, vol. 281, no. 3, pp. 766-777, 2014.

[25] X. Cui, Y. Luo, C. Li, Y. Li, and Z. Wang, "Changes of intracellular $\mathrm{Ca}^{2+}$ in quercetin-induced autophagy progression," Acta Biochimica et Biophysica Sinica, vol. 47, no. 11, pp. 908-914, 2015.

[26] H. S. Seo, J. M. Ku, H.-S. Choi et al., "Quercetin induces caspase-dependent extrinsic apoptosis through inhibition of signal transducer and activator of transcription 3 signaling in HER2-overexpressing BT-474 breast cancer cells," Oncology Reports, vol. 36, no. 1, pp. 31-42, 2016.

[27] M. Granato, C. Rizzello, M. S. Gilardini Montani et al., "Quercetin induces apoptosis and autophagy in primary effusion lymphoma cells by inhibiting PI3K/AKT/mTOR and STAT3 signaling pathways," Journal of Nutritional Biochemistry, vol. 41, pp. 124-136, 2017.

[28] H. Kim, J. Y. Moon, K. S. Ahn, and S. K. Cho, "Quercetin induces mitochondrial mediated apoptosis and protective autophagy in human glioblastoma U373MG cells," Oxidative Medicine and Cellular Longevity, vol. 2013, Article ID 596496, 10 pages, 2013.

[29] T. F. Tsai, T. S. Hwang, J. F. Lin et al., "Suppression of quercetin-induced autophagy enhances cytotoxicity through elevating apoptotic cell death in human bladder cancer cells," Urological Science, vol. 30, no. 2, pp. 58-66, 2019.

[30] D. H. Park, S. T. Lee, D. Y. Jun et al., "Comparative evaluation of antioxidant activities of ethanol extracts and their solvent fractions obtained from selected miscellaneous cereal grains," Journal of Life Science, vol. 24, no. 1, pp. 26-38, 2014.
[31] K. Y. Kim, W. Y. Jang, J. Y. Lee et al., "Kaempferol activates G2-checkpoint of the cell cycle resulting in G2-arrest and mitochondria-dependent apoptosis in human acute leukemia Jurkat T cells," Journal of Microbiology and Biotechnology, vol. 26, no. 2, pp. 287-294, 2016.

[32] N. Zamzami, P. Marchetti, M. Castedo et al., "Reduction in mitochondrial potential constitutes an early irreversible step of programmed lymphocyte death in vivo," Journal of Experimental Medicine, vol. 181, no. 5, pp. 1661-1672, 1995.

[33] P. X. Petit, H. Lecoeur, E. Zorn, C. Dauguet, B. Mignotte, and M. L. Gougeon, "Alterations in mitochondrial structure and function are early events of dexamethasone-induced thymocyte apoptosis," Journal of Cell Biology, vol. 130, no. 1, pp. 157-167, 1995.

[34] C. R. Han, D. Y. Jun, Y. H. Kim, J. Y. Lee, and Y. H. Kim, "Prometaphase arrest-dependent phosphorylation of Bcl-2 family proteins and activation of mitochondrial apoptotic pathway are associated with $17 \alpha$-estradiol-induced apoptosis in human Jurkat T cells," Biochimica et Biophysica Acta (BBA) - Molecular Cell Research, vol. 1833, no. 10, pp. 2220-2232, 2013.

[35] N. E. Rainey, A. Saric, A. Leberre et al., "Synergistic cellular effects including mitochondrial destabilization, autophagy and apoptosis following low-level exposure to a mixture of lipophilic persistent organic pollutants," Scientific Reports, vol. 7, no. 1, 2017.

[36] K. Y. Kim, S. K. Hwang, S. Y. Park, M. J. Kim, D. Y. Jun, and Y. H. Kim, "l-Serine protects mouse hippocampal neuronal HT22 cells against oxidative stress-mediated mitochondrial damage and apoptotic cell death," Free Radical Biology \& Medicine, vol. 141, pp. 447-460, 2019.

[37] C. Riccardi and I. Nicoletti, "Analysis of apoptosis by propidium iodide staining and flow cytometry," Nature Protocols, vol. 1, no. 3, pp. 1458-1461, 2006.

[38] H. Okada and T. W. Mak, "Pathways of apoptotic and nonapoptotic death in tumour cells," Nature Reviews Cancer, vol. 4, no. 8, pp. 592-603, 2004.

[39] J. F. Abrahamsen, A. M. Bakken, O. Bruserud, and B. T. Gjertsen, "Flow cytometric measurement of apoptosis and necrosis in cryopreserved PBPC concentrates from patients with malignant diseases," Bone Marrow Transplantation, vol. 29, no. 2, pp. 165-171, 2002.

[40] P. Li, D. Nijhawan, I. Budihardjo et al., "Cytochrome $c$ and dATP-dependent formation of Apaf-1/caspase- 9 complex initiates an apoptotic protease cascade," Cell, vol. 91, no. 4, pp. 479-489, 1997.

[41] D. Westphal, G. Dewson, P. E. Czabotar, and R. M. Kluck, "Molecular biology of Bax and Bak activation and action," Biochimica et Biophysica Acta (BBA) - Molecular Cell Research, vol. 1813, no. 4, pp. 521-531, 2011.

[42] G. Kroemer, L. Galluzzi, and C. Brenner, "Mitochondrial membrane permeabilization in cell death," Physiological Reviews, vol. 87, no. 1, pp. 99-163, 2007.

[43] H. Li, H. Zhu, C. J. Xu, and J. Yuan, "Cleavage of Bid by caspase 8 mediates the mitochondrial damage in the Fas pathway of apoptosis," Cell, vol. 94, no. 4, pp. 491-501, 1998.

[44] S. Desagher, A. Osen-Sand, A. Nichols et al., "Bid-induced conformational change of Bax is responsible for mitochondrial cytochrome $c$ release during apoptosis," Journal of Cell Biology, vol. 144, no. 5, pp. 891-901, 1999. 
[45] A. Hirao, Y. Y. Kong, S. Matsuoka et al., "DNA damageinduced activation of p53 by the checkpoint kinase Chk2," Science, vol. 287, no. 5459, pp. 1824-1827, 2000.

[46] J. Smith, L. M. Tho, N. Xu, and D. A. Gillespie, "The ATMChk2 and ATR-Chk1 pathways in DNA damage signaling and cancer," Advances in Cancer Research, vol. 108, pp. 73-112, 2010.

[47] G. Jacquemin, V. Granci, A. S. Gallouet et al., "Quercetinmediated Mcl-1 and survivin downregulation restores TRAIL-induced apoptosis in non-Hodgkin's lymphoma B cells," Haematologica, vol. 97, no. 1, pp. 38-46, 2012.

[48] C. M. Liu, J. Q. Ma, and Y. Z. Sun, "Quercetin protects the rat kidney against oxidative stress-mediated DNA damage and apoptosis induced by lead," Environmental Toxicology and Pharmacology, vol. 30, no. 3, pp. 264-271, 2010.

[49] X. Zhu, N. Li, Y. Wang et al., "Protective effects of quercetin on UVB irradiation-induced cytotoxicity through ROS clearance in keratinocyte cells," Oncology Reports, vol. 37, no. 1, pp. 209-218, 2017.

[50] Y. F. Chang, C. W. Chi, and J. J. Wang, "Reactive oxygen species production is involved in quercetin-induced apoptosis in human hepatoma cells," Nutrition and Cancer, vol. 55, no. 2, pp. 201-209, 2006.

[51] W. J. Lee, M. Hsiao, J. L. Chang et al., "Quercetin induces mitochondrial-derived apoptosis via reactive oxygen speciesmediated ERK activation in HL-60 leukemia cells and xenograft," Archives of Toxicology, vol. 89, no. 7, pp. 1103-1117, 2015.

[52] L. Chen, T. Liu, A. Tran et al., "OPA1 mutation and late-onset cardiomyopathy: mitochondrial dysfunction and mtDNA instability," Journal of the American Heart Association, vol. 1, no. 5, p. e003012, 2012.

[53] W. K. E. Ip, N. Hoshi, D. S. Shouval, S. Snapper, and R. Medzhitov, "Anti-inflammatory effect of IL-10 mediated by metabolic reprogramming of macrophages," Science, vol. 356, no. 6337, pp. 513-519, 2017.

[54] R. M. Kluck, E. Bossy-Wetzel, D. R. Green, and D. D. Newmeyer, "The release of cytochrome $c$ from mitochondria: a primary site for $\mathrm{Bcl}-2$ regulation of apoptosis," Science, vol. 275, no. 5303, pp. 1132-1136, 1997.

[55] J. E. Chipuk and D. R. Green, "How do Bcl-2 proteins induce mitochondrial outer membrane permeabilization?," Trends in Cell Biology, vol. 18, no. 4, pp. 157-164, 2008.

[56] A. B. Parrish, C. D. Freel, and S. Kornbluth, "Cellular mechanisms controlling caspase activation and function," Cold Spring Harbor Perspectives in Biology, vol. 5, no. 6, 2013.

[57] D. Sohn, K. Schulze-Osthoff, and R. U. Janicke, "Caspase-8 can be activated by interchain proteolysis without receptortriggered dimerization during drug-induced apoptosis," Journal of Biological Chemistry, vol. 280, no. 7, pp. 5267-5273, 2005.

[58] H. J. Woo, D. Y. Jun, J. Y. Lee, M. H. Woo, C. H. Yang, and Y. H. Kim, "Apoptogenic activity of $2 \alpha, 3 \alpha$-dihydroxyurs-12ene-28-oic acid from Prunella vulgaris var. lilacina is mediated via mitochondria-dependent activation of caspase cascade regulated by Bcl-2 in human acute leukemia Jurkat T cells," Journal of Ethnopharmacology, vol. 135, no. 3, pp. 626-635, 2011.

[59] B. Laukens, C. Jennewein, B. Schenk et al., "Smac mimetic bypasses apoptosis resistance in FADD- or caspase-8deficient cells by priming for tumor necrosis factor $\alpha$-induced necroptosis," Neoplasia, vol. 13, no. 10, pp. 971-IN29, 2011.
[60] R. Mathew, V. Karantza-Wadsworth, and E. White, "Role of autophagy in cancer," Nature Reviews Cancer, vol. 7, no. 12, pp. 961-967, 2007.

[61] Z. J. Yang, C. E. Chee, S. Huang, and F. A. Sinicrope, "The role of autophagy in cancer: therapeutic implications," Molecular Cancer Therapeutics, vol. 10, no. 9, pp. 1533-1541, 2011.

[62] K. Wang, R. Liu, J. Li et al., "Quercetin induces protective autophagy in gastric cancer cells: involvement of Akt-mTORand hypoxia-induced factor $1 \alpha$-mediated signaling," Autophagy, vol. 7, no. 9, pp. 966-978, 2011.

[63] Y. Wang, W. Zhang, Q. Lv, J. Zhang, and D. Zhu, "The critical role of quercetin in autophagy and apoptosis in HeLa cells," Tumour Biology, vol. 37, no. 1, pp. 925-929, 2016.

[64] Y. Feng, D. He, Z. Yao, and D. J. Klionsky, "The machinery of macroautophagy," Cell Research, vol. 24, no. 1, pp. 24-41, 2014.

[65] S. R. Datta, H. Dudek, X. Tao et al., "Akt phosphorylation of BAD couples survival signals to the cell-intrinsic death machinery," Cell, vol. 91, no. 2, pp. 231-241, 1997.

[66] L. del Peso, M. Gonzalez-Garcia, C. Page, R. Herrera, and G. Nunez, "Interleukin-3-induced phosphorylation of BAD through the protein kinase Akt," Science, vol. 278, no. 5338, pp. 687-689, 1997.

[67] Y. P. Yang, L. F. Hu, H. F. Zheng et al., "Application and interpretation of current autophagy inhibitors and activators," Acta Pharmacologica Sinica, vol. 34, no. 5, pp. 625-635, 2013.

[68] X. Liu, K. Sun, H. Wang, and Y. Dai, "Inhibition of autophagy by chloroquine enhances the antitumor efficacy of sorafenib in glioblastoma," Cellular and Molecular Neurobiology, vol. 36, no. 7, pp. 1197-1208, 2016. 


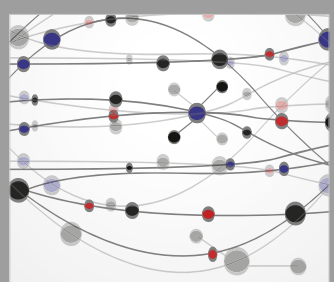

The Scientific World Journal


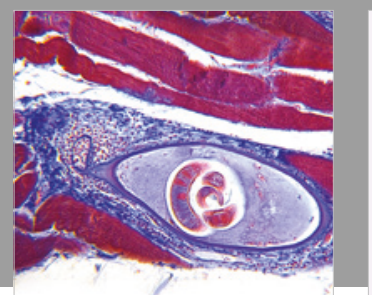

Gastroenterology Research and Practice

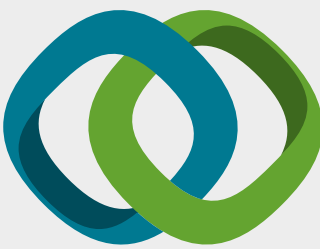

\section{Hindawi}

Submit your manuscripts at

www.hindawi.com
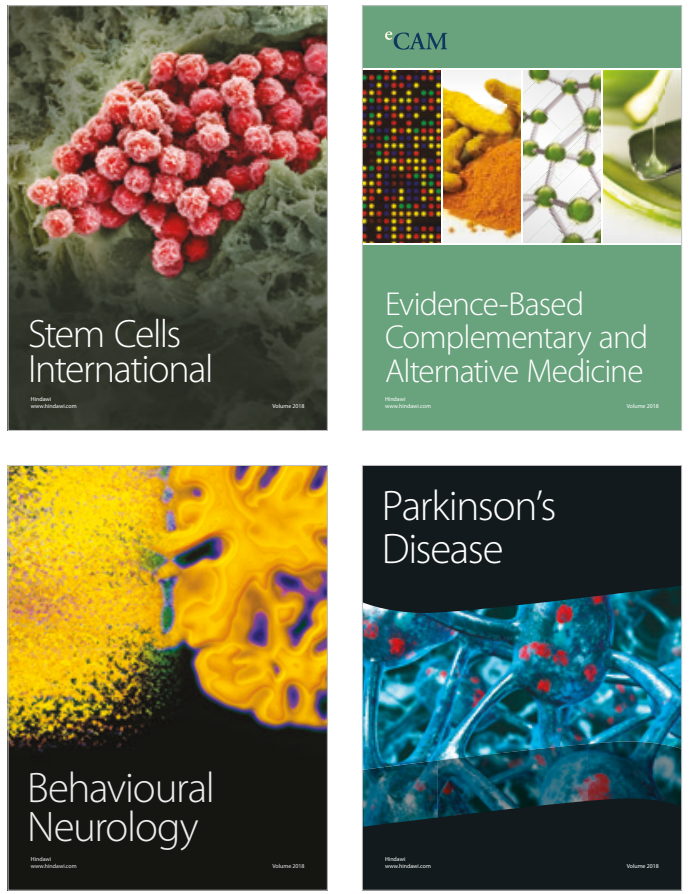

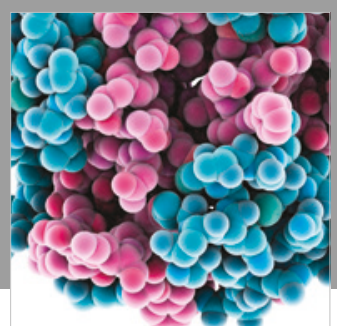

ournal of

Diabetes Research

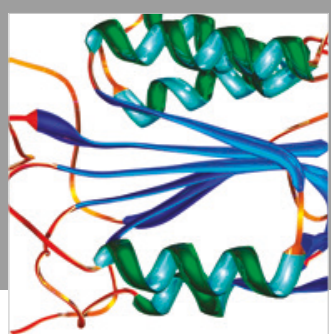

Disease Markers
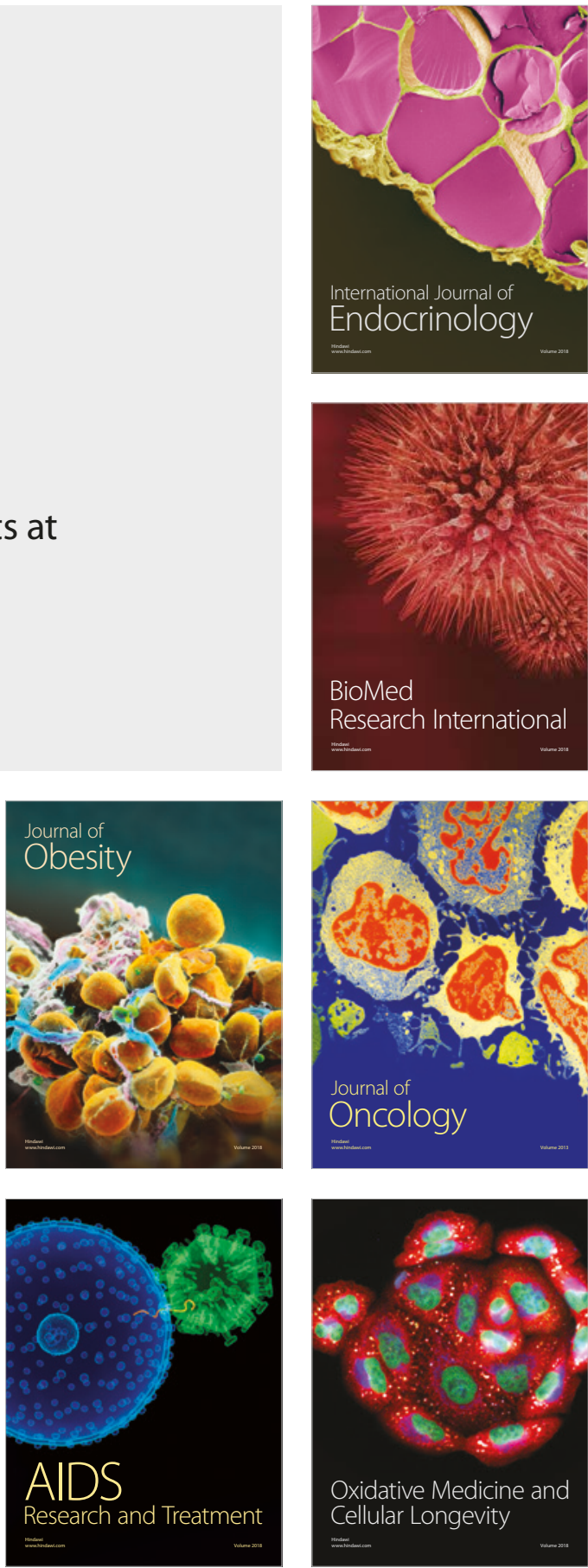